\title{
Eigenvalue hypothesis for multistrand braids
}

\author{
Saswati Dhara, ${ }^{1}$ A. Mironov, ${ }^{2,3,4}$ A. Morozov, ${ }^{2,3,4}$ An. Morozov, ${ }^{3,4}$ P. Ramadevi, ${ }^{1}$ Vivek Kumar Singh, ${ }^{1}$ and A. Sleptsov ${ }^{3,45}$ \\ ${ }^{1}$ Department of Physics, Indian Institute of Technology Bombay, Mumbai 400076, India \\ ${ }^{2}$ Lebedev Physics Institute, Moscow 119991, Russia \\ ${ }^{3}$ ITEP, Moscow 117218, Russia \\ ${ }^{4}$ Institute for Information Transmission Problems, Moscow 127994, Russia \\ ${ }^{5}$ Laboratory of Quantum Topology, Chelyabinsk State University, Chelyabinsk 454001, Russia
}

(Received 10 April 2018; published 21 June 2018)

\begin{abstract}
Computing polynomial form of the colored HOMFLY-PT for nonarborescent knots obtained from three or more strand braids is still an open problem. One of the efficient methods suggested for the three-strand braids relies on the eigenvalue hypothesis which uses the Yang-Baxter equation to express the answer through the eigenvalues of the $\mathcal{R}$ matrix. In this paper, we generalize the hypothesis to higher number of strands in the braid where commuting relations of non-neighboring $\mathcal{R}$ matrices are also incorporated. By solving these equations, we determine the explicit form for $\mathcal{R}$ matrices and the inclusive Racah matrices in terms of braiding eigenvalues (for matrices of size up to 6 by 6). For comparison, we briefly discuss the highest weight method for four-strand braids carrying fundamental and symmetric rank two $S U_{q}(N)$ representation. Specifically, we present all the inclusive Racah matrices for representation [2] and compare with the matrices obtained from eigenvalue hypothesis.
\end{abstract}

DOI: 10.1103/PhysRevD.97.126015

\section{INTRODUCTION}

Classification of knots is one of the most challenging research problems. The well-known Jones, HOMFLY-PT, and Kauffman polynomials [1,2] can distinguish many inequivalent knots but not all knots. Witten's pioneering work [3] involving Chern-Simons field theory [4] and Jones' polynomials suggested that the generalized knot invariants can be computed for any knot $\mathcal{K}$ carrying arbitrary representation $R$ of any gauge group $\mathcal{G}$. They are referred to as colored knot invariants $H_{R}^{\mathcal{K}}$ which are supposed to give a pool of data to attempt the famous challenging problem of the "classification of knots."

The methodology of writing the knot invariants is straightforward and involves braiding eigenvalues and Racah matrices. However, the polynomial form of such invariants can be determined only if we know the Racah matrices. In fact, the Racah matrices are fully known [5] only for $S U_{q}(2)$, enabling the evaluation of the colored Jones polynomial for any knot. Recently, from the colored HOMFLY-PT for twist knots [6] and pretzel knots [7] (using the evolution method [8-10] in the latter case), the closed form expression for Racah matrices involving any $S U_{q}(N)$ symmetric representation was

Published by the American Physical Society under the terms of the Creative Commons Attribution 4.0 International license. Further distribution of this work must maintain attribution to the author(s) and the published article's title, journal citation, and DOI. Funded by SCOAP ${ }^{3}$. conjectured $[7,11,12]$ Subsequently, colored HOMFLY-PT for arborescent knots $[2,13]$ carrying symmetric representations was computable $[6,7,14,15]$ In addition, colored HOMFLY-PT for some rectangular [16] and nonrectangular representations $[17,18]$ of $S U_{q}(N)$ have been obtained for arborescent knots. It is still an open problem to compute colored HOMFLY-PT for any nonarborescent knot carrying symmetric and other representations.

Based on the Reshetikhin-Turaev (RT) approach [19] and its variants, new methods have been devised to obtain colored invariants for nonarborescent knots from closure of three or more strand braids. In Ref. [20], a universal construction for knots in the fundamental representation was suggested but has not been generalized to higher representations. Another approach, used in Refs. [21-25] involves calculations of the highest weight vector for various representations which becomes computationally tedious as we increase the number of braids. We refer the reader to see the papers $[26,27]$ where some nonarborescent knot invariants are presented from generalization of the method [18] Even though these approaches are straightforward, the evaluation process becomes cumbersome. Another powerful method called eigenvalue hypothesis was suggested in Ref. [28] In fact, we will focus on the essential details of the eigenvalue hypothesis which will provide some inclusive Racah matrices to simplify the tedious highest weight approach.

The eigenvalues hypothesis claims that the inclusive Racah matrix is fully determined by the eigenvalues of the $\mathcal{R}$ matrix. Using the suggested hypothesis for the threestrand case [28], the inclusive Racah matrices up to size 
$5 \times 5$ in terms of the $\mathcal{R}$-matrix eigenvalues have been guessed for $S U_{q}(2)^{1}$ and later were shown to work for arbitrary $S U_{q}(N)$. Subsequently, the $6 \times 6$ inclusive Racah matrix was calculated in terms of the eigenvalues [29] using the Vögel universality hypothesis of Chern-Simons theory $[30,31]$. These conjectured matrices have been independently checked. [32] Let us briefly discuss the construction of the inclusive Racah matrix [21] for the three-strand braid. This will set the stage to go to four or more strand braids. For the three-strand braid carrying $S U_{q}(N)$ representations $V_{1}, V_{2}, V_{3}$, it is well known that the Racah matrix $U_{i j}$ (originally introduced by G. Racah and E. P. Wigner) is the matrix that intertwines the maps:

$$
\left(V_{1} \otimes V_{2}\right) \otimes V_{3} \longrightarrow Q \quad \text { and } \quad V_{1} \otimes\left(V_{2} \otimes V_{3}\right) \longrightarrow Q
$$

The matrix indices of $U_{i j}$ are enumerated by the representations from $V_{1} \otimes V_{2}=\oplus_{i} T_{i}$ (the first index) and from $V_{2} \otimes V_{3}=\oplus_{j} T_{j}$ (the second index). Since we are interested in the construction of knots from braids, we need to consider $V_{1}=V_{2}=V_{3}=V$. In fact, $U_{i j}$ are the matrices that relate the braiding matrix $\left(\mathcal{R}_{1}\right)$ acting on the first two strands and the braiding matrix $\left(\mathcal{R}_{2}\right)$ on the second and third strands in the three-strand braid. Note that $U_{i j}$ are referred to as the inclusive Racah matrix when $V \neq Q$ and the exclusive Racah matrix when $V=Q$.

Whatever the representations $V$ and $Q$ are, if the Racah matrix has size $k \times k$, it is expressed through $k$ eigenvalues of the $\mathcal{R}$ matrix $\mathcal{R}: V \otimes V \rightarrow V \otimes V$. These eigenvalues are very simple: $\pm q^{C_{2}(X)}$, where $C_{2}(X)$ is the eigenvalue of the second Casimir operator in representation $X \in V \otimes V$. The evaluation of $U_{i j}$ is governed by the Yang-Baxter equation:

$$
\mathcal{R}_{1} \mathcal{R}_{2} \mathcal{R}_{1}=\mathcal{R}_{2} \mathcal{R}_{1} \mathcal{R}_{2}
$$

In particular, one can diagonalize $\mathcal{R}_{1}$ and take $\mathcal{R}_{2}=$ $U \mathcal{R}_{1} U^{\dagger}$. Then (1) relates the Racah matrix $U$ to the eigenvalues of $\mathcal{R}_{1}$. In principle, for various diagonal $k \times k$ $\mathcal{R}_{1}$, the elements of the $k \times k$ Racah matrix $U_{i j}$ must be determined using the above equation. So far, $U$ up to the size $5 \times 5[28,33]$ and for the size $6 \times 6[29]$ has been obtained.

Going beyond three-strand braids has not been discussed within the context of eigenvalue hypothesis. In this paper, we demonstrate that the eigenvalue hypothesis is still true for the case of multistrand braids. We illustrate it in the example of four-strand braids (i.e., for the maps $V^{\otimes 4} \rightarrow Q$ ). In this case, there are three $\mathcal{R}_{i=1,2,3}$ matrices and two unitary matrices $U, W_{1}$. Hereafter, we call all these unitary matrices as inclusive Racah matrices even though they are

\footnotetext{
${ }^{1}$ It works in the following way: let us fix $V$ and $Q$ to be the spin $j$ and $3 j-k+1$ representations of $S U_{q}(2)$, respectively. Then, the inclusive Racah matrix $U_{k \times k}(j)$ for $V^{\otimes 3} \rightarrow Q$ has size $k \times k$, and the $k$ eigenvalues of the $\mathcal{R}$ matrix, $\lambda_{i}(j), i=1, \ldots, k$ are parametrized by $j$.
}

more general matrices that relate the braiding matrices ( $\mathcal{R}_{i}$ 's) in the multistrand braids. Note that the matrix $U$ that makes the rotation of $\mathcal{R}_{1}$ to $\mathcal{R}_{2}$ is still the same and is determined by the Yang-Baxter equation (1). The rotation to $\mathcal{R}_{3}=U W_{1} U \mathcal{R}_{1} U^{\dagger} W_{1}^{\dagger} U^{\dagger}$ involves also the $W_{1}$ matrix besides $U$, which is determined from the requirement that $\mathcal{R}_{1}$ and $\mathcal{R}_{3}$ commute:

$$
\left[\mathcal{R}_{1}, \mathcal{R}_{3}\right]=0 .
$$

This property comes, in fact, from the braid-group relations which $\mathcal{R}$ matrices should satisfy. We solved this equation for $W_{1}$ up to matrices of size $6 \times 6$ and checked that the result for the Racah matrices obtained this way coincides with the Racah matrices evaluated by the highest weight method.

With the above discussions for three- and four-strand braids, we can now formulate the eigenvalue hypothesis for the generic $n$-strand braid:

Extended eigenvalue hypothesis. The Racah matrices defining $\mathcal{R}_{2}$ are determined by the Yang-Baxter equation (1), while the remaining Racah matrices defining $\mathcal{R}_{i}, i \geq 3$ are determined by commuting with all nonneighbor $\mathcal{R}$ matrices [parametrized like $\mathcal{R}_{4}$ in (11)]:

$$
\mathcal{R}_{i} \mathcal{R}_{j}=\mathcal{R}_{j} \mathcal{R}_{i}, \quad|j-i| \neq 1 .
$$

In other words, according to this hypothesis, if one makes the $\mathcal{R}_{1}$ matrix diagonal with all the eigenvalues different from each other, then all other matrices are uniquely defined. Therefore, they provide some particular representation of the braid group.

The paper is organized as follows. In Sec. II, we discuss general properties of $\mathcal{R}$ matrices involved in the ReshetikhinTuraev approach. In Sec. III, we outline the basics of eigenvalue hypothesis with Sec. III B presenting old results from [28] for the three-strand case, and Sec. III C presenting new results for the four-strand eigenvalue hypothesis. In Sec. IV, we explain the highest weight method, which allows us to evaluate the Racah matrices. In particular, we indicate the calculation of $U$ in the three-strand braid for a specific representation. These matrix elements agree with those of the eigenvalue hypothesis in Sec. III up to the \pm sign. We have presented $U$ and $W_{1}$ for $R=$ [1] in Sec. VA few of the unitary matrices for $R=$ [2] are given in Sec. V B. Summary and open questions are posed in the concluding Sec. VI. In Appendix A, we have placed the remaining $U$ and $W_{1}$ for $k \times k$ where $k>3$ for representation $R=$ [2]. Appendix B contains [2]-colored HOMFLY-PT for a few arborescent knots and all nonarborescent knots up to 10-crossings obtained from four-strand braids. We will update these polynomials in our web site [34].

\section{II. $\mathcal{R}$ MATRICES}

One of the most useful approaches to calculate knot polynomials and the one relevant to the subject of the paper 
is the so-called Reshetikhin-Turaev (RT) approach [19, 21-25,35,36]. This approach deals with the braid representation of the knot. Each crossing in the braid corresponds to a particular $\mathcal{R}$ matrix. Then the knot polynomial is presented as a character expansion

$$
H_{Y}^{K}=\sum_{Q \vdash Y^{\otimes m}} S_{Q}^{*} C_{Q}
$$

where the sum is over all irreducible representations in the product or representations corresponding to individual components, $m$ is the number of strands, $Y$ is the representation on each strand (in this paper, we consider only knots, but most of the formulas in this section can be extended also to links), $C_{Q}$ is the trace of the product of all $\mathcal{R}$ matrices along the braid in the linear space of all intertwining operators $Y^{\otimes m} \rightarrow Q$, and $S_{Q}^{*}$ is the quantum dimension of the representation $Q$.

Let us denote through $\mathcal{R}_{i}$ the $\mathcal{R}$ matrix corresponding to the crossing between the $i$ th and $(i+1)$ th braid. This matrix is defined by the following three properties:

(i) The property of any $\mathcal{R}_{i}$, its characteristic equation ${ }^{2}$ :

$$
\prod_{j}\left(\mathcal{R}_{i}-\lambda_{j}\right)=0 .
$$

(ii) The Yang-Baxter equation, which, in the case of a braid, has the following form:

$$
\mathcal{R}_{i} \mathcal{R}_{i+1} \mathcal{R}_{i}=\mathcal{R}_{i+1} \mathcal{R}_{i} \mathcal{R}_{i+1} .
$$

(iii) The commutativity of non-neighbor $\mathcal{R}$ matrices:

$$
\mathcal{R}_{i} \mathcal{R}_{j}=\mathcal{R}_{j} \mathcal{R}_{i}, \quad i \neq j \pm 1
$$

\section{A. Racah matrices}

Since all $\mathcal{R}$ matrices in the braid have the same sets of eigenvalues they are related by rotation matrices which are, in fact, the inclusive Racah matrices. These inclusive Racah matrices possess a very special structure.

Let us choose $\mathcal{R}_{1}$ to be diagonal. $\mathcal{R}_{1}$ can be associated with the following way of putting parentheses in the product of representations: $(\ldots((Y \otimes Y) \otimes Y) \otimes . . Y)$. Then, $\mathcal{R}_{2}$ would correspond to the other way: $(\ldots(Y \otimes$ $(Y \otimes Y)) \otimes . . Y)$. The rotation from one way to another can be described by the following trees of representations:

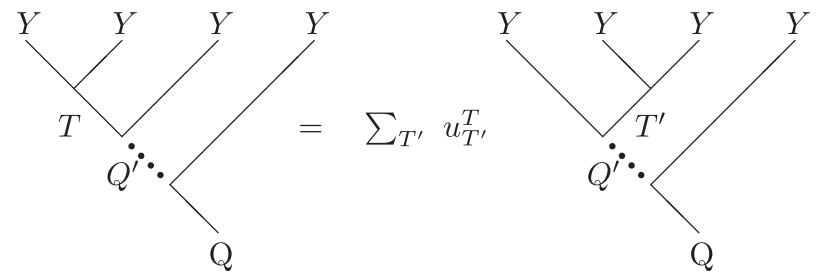

$u_{T^{\prime}}^{T}$ are elements of the matrix $U$ corresponding to the Racah coefficient $\left[\begin{array}{ccc}Y & Y & T \\ Y & Q^{\prime} & T\end{array}\right]$. $\mathcal{R}_{2}$ is then defined as

$$
\mathcal{R}_{2}=U \mathcal{R}_{1} U^{\dagger} .
$$

If one studies three-strand braids then $Q^{\prime}=Q$. However, for larger number of strands, they are not equal. Thus, from the form of this inclusive Racah matrix it is obvious that only the elements of the matrix corresponding to the same $Q^{\prime}$ are nonzero. Hence, such $U$ has a block diagonal form with different blocks corresponding to different $Q^{\prime}$.

The third matrix, $\mathcal{R}_{3}$ corresponds to the following product of representations: $(\ldots(Y \otimes(Y \otimes(Y \otimes Y))) \otimes . . Y)$. Thus, the transition from $\mathcal{R}_{1}$ to $\mathcal{R}_{3}$ should be made through the chain of trees

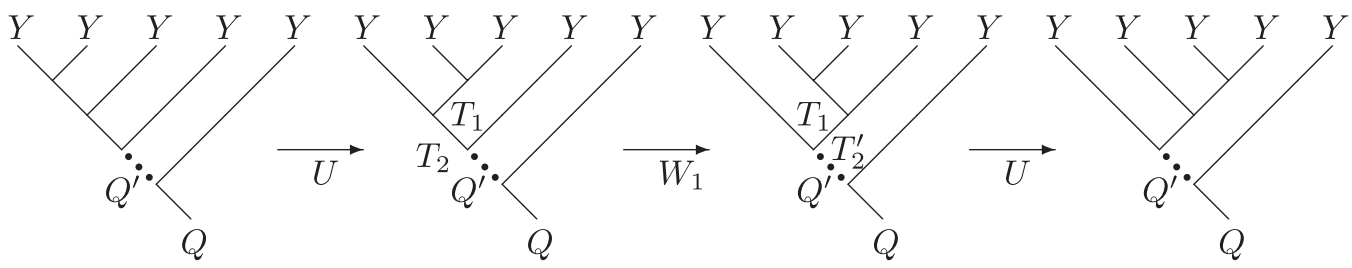

\footnotetext{
${ }^{2}$ Since the braid $\mathcal{R}$ matrix acting on $Y \otimes Y$ commutes with the coproduct [37], it is diagonal in the basis of irreps $Q \in Y \otimes Y$. Its eigenvalues are all expressed through the basic quantity

$$
\varkappa_{Q}=1 / 2 \sum_{i} Q_{i}\left(Q_{i}+1-2 i\right)
$$

associated with the Young diagram $Q$ with lines $Q_{1} \geq Q_{2} \ldots \geq 0$. Hereafter, we don't differ between the representation $Q$ and the Young diagram that describes $Q$. The eigenvalues then are given by the formula

$$
\lambda_{Q}=\epsilon_{Q} q^{\varkappa_{Q}}
$$

where the sign factors $\epsilon_{Q}= \pm 1$ depend on whether $Q$ lies in the symmetric $(+1)$ or antisymmetric $(-1)$ square of $Y$; see [23,27].
} 
The first and the last matrices are actually the same matrices as appeared above. But for relating $\mathcal{R}_{3}$ with $\mathcal{R}_{1}$ a new matrix $W_{1}$ is needed. This matrix corresponds to the Racah coefficient $\left[\begin{array}{lll}Y & T_{1} & T_{2} \\ Y & Q^{\prime} & T_{2}^{\prime}\end{array}\right] . \mathcal{R}_{3}$ is then defined as

$$
\mathcal{R}_{3}=U W_{1} U \mathcal{R}_{1} U^{\dagger} W_{1}^{\dagger} U^{\dagger}
$$

Again, similarly to the case of $U$, the only nonzero elements of matrix $W_{1}$ correspond to the coinciding $T_{1}$ and coinciding $Q^{\prime}$. This leads to a very interesting property of the matrix $W_{1}$. Since the eigenvalues of the diagonal $\mathcal{R}$ matrix are defined by the representation $T_{1}, W_{1}$ commutes with the diagonal $\mathcal{R}$ matrix.

Similarly, this structure can be continued to further $\mathcal{R}$ matrices, e.g., for $\mathcal{R}_{4}$ :

$\mathcal{R}_{4}=U W_{1} U W_{2} U W_{1} U \mathcal{R}_{1} U^{\dagger} W_{1}^{\dagger} U^{\dagger} W_{2}^{\dagger} U^{\dagger} W_{1}^{\dagger} U^{\dagger}$.

$W_{2}$ then again possesses a block structure. This block structure can be described by paths from the initial representation $Y$ to the final representation $Q$ [21]. This is a generalization of the statements made about the block structure of matrices $U$ and $W_{1}$. If one defines the representation $Q$ as coming from the following sequence of representations

$$
Y \rightarrow T_{1} \rightarrow T_{2} \rightarrow T_{3} \rightarrow \ldots \rightarrow Q,
$$

then the matrix $W_{i}$ has nonzero elements only for the final representations $Q$ corresponding to the same $T_{1}, T_{2}, . . T_{i}, T_{i+2}, \ldots$ This allows one to define the block structure of any Racah matrix $W_{i}$.

Let us discuss some particular example of this path and block structure, e.g., $[2]^{\otimes 4}=[5,3]$. This is a four-strand case; thus, only the Racah matrices $U$ and $W_{1}$ appear. The multiplicity of representation [5,3] is equal to 6 which means 6 possible paths:

$$
\begin{aligned}
& \text { 1. }[2] \rightarrow[4] \rightarrow[5,1] \rightarrow[5,3] \\
& \text { 2. }[2] \rightarrow[4] \rightarrow[4,2] \rightarrow[5,3] \\
& \text { 3. }[2] \rightarrow[3,1] \rightarrow[5,1] \rightarrow[5,3] \\
& \text { 4. }[2] \rightarrow[3,1] \rightarrow[4,2] \rightarrow[5,3] \\
& \text { 5. }[2] \rightarrow[3,1] \rightarrow[3,3] \rightarrow[5,3] \\
& \text { 6. }[2] \rightarrow[2,2] \rightarrow[4,2] \rightarrow[5,3]
\end{aligned}
$$

The matrix $U$ then has three blocks. The first one mixes paths 1 and 3, the second one mixes paths 2, 4 and 6 and the third one corresponds only to path 5 . Then the matrix $W_{1}$ also has three blocks. The first one mixes paths 1 and 2, the second one mixes paths 3,4 and 5 and the third one corresponds to path 6 .

\section{EIGENVALUE HYPOTHESIS}

In this section, we discuss how the properties of $\mathcal{R}$ matrices define their form and how the eigenvalue hypothesis appear from these properties.

\section{A. Two-strand case}

In the two-strand case, there exists only one $\mathcal{R}$ matrix and only one property of the three discussed is important, namely, the characteristic equation (4). This property defines eigenvalues of the $\mathcal{R}$ matrix. In fact, this property is essentially two-strand. This means that even if we study larger number of strands it does not give any further information and includes only one $\mathcal{R}$ matrix acting on two adjacent strands.

\section{B. Three-strand case}

In the three-strand case, there are two $\mathcal{R}$ matrices, $\mathcal{R}_{1}$ and $\mathcal{R}_{2}$, related by one Racah matrix $U$ :

$$
\mathcal{R}_{2}=U \mathcal{R}_{1} U^{\dagger}
$$

As already explained in the previous subsection, (4) does not give any new information about these $\mathcal{R}$ matrices and only describes that they have the same eigenvalues. However, the Yang-Baxter equation (5) is of great importance here. In terms of the Racah matrix $U$ this equation looks like

$$
\mathcal{R}_{1} U \mathcal{R}_{1} U^{\dagger} \mathcal{R}_{1}=U \mathcal{R}_{1} U^{\dagger} \mathcal{R}_{1} U \mathcal{R}_{1} U^{\dagger}
$$

Incorporating unitarity of $U\left(U U^{\dagger}=1\right)$ into the above equation one comes to the eigenvalue hypothesis. For the matrices of the size up to $6 \times 6$, there is a unique solution for matrix $U$ if the $\mathcal{R}$ matrix is chosen to be diagonal. Strictly speaking, there are several solutions differing by inessential sign changes, and, at some special values of the eigenvalues, more solutions can also emerge. For $2 \times 2$ matrices the unique solution to (15) in the case of two generic eigenvalues looks like:

$$
U=\left(\begin{array}{cc}
\frac{\sqrt{-\lambda_{1} \lambda_{2}}}{\lambda_{1}-\lambda_{2}} & \frac{\sqrt{\lambda_{1}^{2}-\lambda_{1} \lambda_{2}+\lambda_{2}^{2}}}{\lambda_{1}-\lambda_{2}} \\
\frac{\sqrt{\lambda_{1}^{2}-\lambda_{1} \lambda_{2}+\lambda_{2}^{2}}}{\lambda_{1}-\lambda_{2}} & \frac{\sqrt{-\lambda_{1} \lambda_{2}}}{\lambda_{1}-\lambda_{2}}
\end{array}\right), \quad \text { for } \mathcal{R}_{1}=\left(\begin{array}{ll}
\lambda_{1} & \\
& \lambda_{2}
\end{array}\right) .
$$

Similar answers can be found for matrices of larger sizes.

As characteristic equation was essentially a two-strand property, the Yang-Baxter equation is a three-strand property and does not give anything new for larger number of strands. This is explained in detail for the four-strand braid in the next subsection. 


\section{Four-strand case}

For the four-strand situation, there are three $\mathcal{R}$ matrices and two inclusive Racah matrices, $U$ and $W_{1}$. Here, the most important is the third property (6).

Suppose all the eigenvalues of $\mathcal{R}$ matrices are different, then the only solution to (6) is to have $\mathcal{R}_{1}=\mathcal{R}_{3}$ and $\mathcal{R}_{2}$ is defined from the first three strands as discussed in Sec. III B.

If some eigenvalues coincide the situation is more interesting. According to Sec. II A, both $U$ and $W_{1}$ have the block-diagonal form. Also $W_{1}$ commutes with $\mathcal{R}_{1}$. If $\mathcal{R}_{1}$ is diagonal, then

$$
\mathcal{R}_{2}=U \mathcal{R}_{1} U^{\dagger}, \quad \mathcal{R}_{3}=U W_{1} U \mathcal{R}_{1} U^{\dagger} W_{1}^{\dagger} U^{\dagger},
$$

on the other hand, if one diagonalizes $\mathcal{R}_{2}=\mathcal{R}$, then $\mathcal{R}_{3}=W_{1} U \mathcal{R}_{2} U^{\dagger} W_{1}^{\dagger}$, and $W_{1}$ commutes with $\mathcal{R}$. Then, the Yang-Baxter equation on $\mathcal{R}_{2}$ and $\mathcal{R}_{3}$ looks like

$$
\mathcal{R}\left(W_{1} U \mathcal{R} U^{\dagger} W_{1}^{\dagger}\right) \mathcal{R}=\left(W_{1} U \mathcal{R} U^{\dagger} W_{1}^{\dagger}\right) \mathcal{R}\left(W_{1} U \mathcal{R} U^{\dagger} W_{1}^{\dagger}\right) .
$$

Since $W_{1}$ commutes with $\mathcal{R}$, this equation transforms into

$$
\mathcal{R} U \mathcal{R} U^{\dagger} \mathcal{R}=U \mathcal{R} U^{\dagger} \mathcal{R} U \mathcal{R} U^{\dagger},
$$

which is automatically satisfied because of the construction in Sec. III B and does not include $W_{1}$. Thus, $W_{1}$ cannot be found from the Yang-Baxter equation, and one needs another equation for the Racah coefficients to find $W_{1}$. This equation comes from the third property (6):

$$
U W_{1} U \mathcal{R} U^{\dagger} W_{1}^{\dagger} U^{\dagger} \mathcal{R}=\mathcal{R} U W_{1} U \mathcal{R} U^{\dagger} W_{1}^{\dagger} U^{\dagger}
$$

$$
U=\left(\begin{array}{ccccc}
1 & & & \\
& \frac{\sqrt{-\lambda_{1} \lambda_{2}}}{\lambda_{1}-\lambda_{2}} & \frac{\sqrt{\lambda_{1}^{2}-\lambda_{1} \lambda_{2}+\lambda_{2}^{2}}}{\lambda_{1}-\lambda_{2}} & & \\
\frac{\sqrt{\lambda_{1}^{2}-\lambda_{1} \lambda_{2}+\lambda_{2}^{2}}}{\lambda_{1}-\lambda_{2}} & -\frac{\sqrt{-\lambda_{1} \lambda_{2}}}{\lambda_{1}-\lambda_{2}} & & \\
& & \frac{\lambda_{1}\left(\lambda_{2}+\lambda_{3}\right)}{\left(\lambda_{1}-\lambda_{3}\right)\left(\lambda_{1}-\lambda_{2}\right)} & \frac{\sqrt{\left(\lambda_{1}^{2}+\lambda_{2} \lambda_{3}\right)\left(\lambda_{2}^{2}+\lambda_{1} \lambda_{3}\right)}}{\left(\lambda_{1}-\lambda_{2}\right) \sqrt{\left(\lambda_{1}-\lambda_{3}\right)\left(\lambda_{3}-\lambda_{2}\right)}} & \frac{\sqrt{\left(\lambda_{1}^{2}+\lambda_{2} \lambda_{3}\right)\left(\lambda_{3}^{2}+\lambda_{1} \lambda_{2}\right)}}{\left(\lambda_{1}-\lambda_{3}\right) \sqrt{\left(\lambda_{1}-\lambda_{2}\right)\left(\lambda_{2}-\lambda_{3}\right)}} \\
& & \frac{\sqrt{\left(\lambda_{1}^{2}+\lambda_{2} \lambda_{3}\right)\left(\lambda_{2}^{2}+\lambda_{1} \lambda_{3}\right)}}{\left(\lambda_{1}-\lambda_{2}\right) \sqrt{\left(\lambda_{1}-\lambda_{3}\right)\left(\lambda_{3}-\lambda_{2}\right)}} & -\frac{\lambda_{2}\left(\lambda_{1}+\lambda_{3}\right)}{\left(\lambda_{1}-\lambda_{2}\right)\left(\lambda_{2}-\lambda_{3}\right)} & \frac{\sqrt{\left(\lambda_{2}^{2}+\lambda_{1} \lambda_{3}\right)\left(\lambda_{3}^{2}+\lambda_{1} \lambda_{2}\right)}}{\left(\lambda_{2}-\lambda_{3}\right) \sqrt{-\left(\lambda_{1}-\lambda_{2}\right)\left(\lambda_{1}-\lambda_{3}\right)}} \\
& \frac{\sqrt{\left(\lambda_{1}^{2}+\lambda_{2} \lambda_{3}\right)\left(\lambda_{3}^{2}+\lambda_{1} \lambda_{2}\right)}}{\left(\lambda_{1}-\lambda_{3}\right) \sqrt{\left(\lambda_{1}-\lambda_{2}\right)\left(\lambda_{2}-\lambda_{3}\right)}} & \frac{\sqrt{\left(\lambda_{2}^{2}+\lambda_{1} \lambda_{3}\right)\left(\lambda_{3}^{2}+\lambda_{1} \lambda_{2}\right)}}{\left(\lambda_{2}-\lambda_{3}\right) \sqrt{-\left(\lambda_{1}-\lambda_{2}\right)\left(\lambda_{1}-\lambda_{3}\right)}} & \frac{\lambda_{3}\left(\lambda_{1}+\lambda_{2}\right)}{\left(\lambda_{1}-\lambda_{3}\right)\left(\lambda_{2}-\lambda_{3}\right)}
\end{array}\right) .
$$

Then from (20), one can find how the matrix $W_{1}$ looks: 


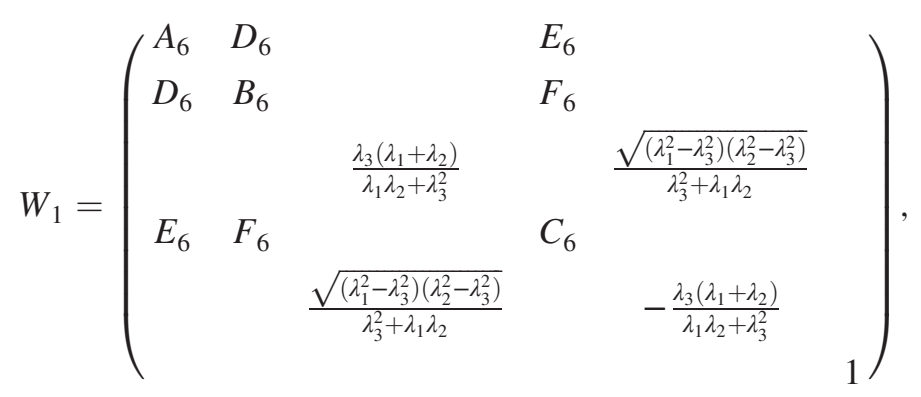

where

$$
\begin{aligned}
& A_{6}=\frac{\lambda_{1}^{2} \lambda_{2}\left(\lambda_{2}+\lambda_{3}\right)}{\left(\lambda_{1}^{2}+\lambda_{2} \lambda_{3}\right)\left(\lambda_{1}^{2}-\lambda_{1} \lambda_{2}+\lambda_{2}^{2}\right)} ; \quad B_{6}=\frac{\lambda_{2}\left(\lambda_{1}^{3}-\lambda_{1}^{2} \lambda_{2}-\lambda_{1}^{2} \lambda_{3}-\text { lambda } \lambda_{3}^{2}+\lambda_{2} \lambda_{3}^{2}-\lambda_{1} \lambda_{2} \lambda_{3}\right)}{\left(\lambda_{3}^{2}+\lambda_{1} \lambda_{2}\right)\left(\lambda_{1}^{2}-\lambda_{1} \lambda_{2}+\lambda_{2}^{2}\right)} ; \\
& C_{6}=\frac{\lambda_{1} \lambda_{3}\left(\lambda_{1}+\lambda_{2}\right)\left(\lambda_{2}+\lambda_{3}\right)}{\left(\lambda_{1}^{2}+\lambda_{2} \lambda_{3}\right)\left(\lambda_{3}^{2}+\lambda_{1} \lambda_{2}\right)} ; \quad D_{6}=\frac{1}{\lambda_{1}^{2}-\lambda_{1} \lambda_{2}+\lambda_{2}^{2}} \sqrt{\frac{\lambda_{1}\left(\lambda_{1}-\lambda_{2}\right)\left(\lambda_{2}+\lambda_{3}\right)\left(\lambda_{2}^{2}+\lambda_{1} \lambda_{3}\right)\left(\lambda_{1}^{3}-\lambda_{2}^{2} \lambda_{3}\right)}{\left(\lambda_{1}^{2}+\lambda_{2} \lambda_{3}\right)\left(\lambda_{3}^{2}+\lambda_{1} \lambda_{2}\right)}} ; \\
& E_{6}=-\frac{\lambda_{1}-\lambda_{3}}{\lambda_{1}^{2}+\lambda_{2} \lambda_{3}} \sqrt{\frac{\lambda_{2}\left(\lambda_{1}+\lambda_{3}\right)\left(\lambda_{1}-\lambda_{2}\right)\left(\lambda_{1}^{3}-\lambda_{2}^{2} \lambda_{3}\right)}{\left(\lambda_{3}^{2}+\lambda_{1} \lambda_{2}\right)\left(\lambda_{1}^{2}-\lambda_{1} \lambda_{2}+\lambda_{2}^{2}\right)}} ; \quad F_{6}=-\frac{\lambda_{1}-\lambda_{3}}{\lambda_{3}^{2}+\lambda_{1} \lambda_{2}} \sqrt{\frac{\lambda_{1} \lambda_{2}\left(\lambda_{1}+\lambda_{3}\right)\left(\lambda_{2}+\lambda_{3}\right)\left(\lambda_{2}^{2}+\operatorname{lambda}_{1} \lambda_{3}\right)}{\left(\lambda_{1}^{2}+\lambda_{2} \lambda_{3}\right)\left(\lambda_{1}^{2}-\lambda_{1} \operatorname{lambda}_{2}+\lambda_{2}^{2}\right)} .}
\end{aligned}
$$

(4) For larger sizes of matrices, the blocks of size $4 \times 4$ appear, and it is rather tedious to find the answers in this case.

The results of this section have been confirmed from the highest weight method (reviewed in Sec. IV) for some representations which are presented in Sec. $\mathrm{V}$ and Appendix A.

\section{HIGHEST WEIGHT METHOD}

In this section, we formally present the highest weight method for any $m$-strand braid carrying an arbitrary representation $Y$ of the quantum group $S U_{q}(N)$.

This method is a systematic procedure which allows one to construct a highest weight vector state. It is based on the manifest action of lowering $T_{i}^{-}$and raising operators $T_{i}^{+}$on representations of $S U_{q}(N)$ [21]:

$$
\begin{array}{rlrl}
T_{i}^{-} V_{i} & =V_{i-1} ; & T_{i}^{+} V_{i-1}=V_{i} . \\
H_{i} V_{i}=+\frac{1}{2} V_{i} ; & H_{i} V_{i-1}=-\frac{1}{2} V_{i-1} .
\end{array}
$$

where $V_{i}$ is an $i$ th vector of the fundamental representation, and $T_{i}^{+}, T_{i}^{-}$and $q^{H_{i}}$ are generators of $S U_{q}(N)$. To generalize this action to higher rank tensors, one has to define a comultiplication $\Delta$ on $S U_{q}(N)$ :

$$
\begin{aligned}
& \Delta\left(T_{i}^{+}\right)=\mathbb{I} \otimes T_{i}^{+}+T_{i}^{+} \otimes q^{-2 H_{i}} \\
& \Delta\left(T_{i}^{-}\right)=q^{2 H_{i}} \otimes T_{i}^{-}+T_{i}^{-} \otimes \mathbb{I} .
\end{aligned}
$$

This extends the action of $T_{i}^{ \pm}$to tensors of any rank. We indicate the highest weight vector for a representation $R$ labeled by Young diagram $\left[\lambda_{1}, \lambda_{2}, \lambda_{3} \ldots \lambda_{l}\right]$ as a sum of $V_{(}(\underbrace{0,0,0}_{\lambda_{1}}, \ldots, \underbrace{1,1,1}_{\lambda_{2}}$, ldots $)$ and their permutations. For example, the highest weight state for $R=[1,1]$ will involve $V_{0,1}$ and $V_{1,0}$. One can construct all the highest weight vectors of the representation by using the lowering and raising operators $T_{i}^{ \pm}$and the comultiplication rule.

We would like to validate the results of inclusive Racah matrices obtained from the eigenvalue hypothesis using the highest weight approach. For definiteness, we take representation $R=[2]$ and construct the highest weight states for $m=3$ and $m=4$ strand braids in the following subsections.

\section{A. Evaluation of vector states for $\boldsymbol{m}=\mathbf{3}$}

Our goal is to evaluate the $U$ matrix corresponding to all irreducible representations in the fusion channel of $[2]^{\otimes 3}$ for the $m=3$ strand braid shown in Eq. (29). One can easily see the two possible fusion trees which give two sets of irreducible representations $Q$ in the final 
channel which are related by a unitary matrix, its size being determined by the multiplicity indicated by the red color:
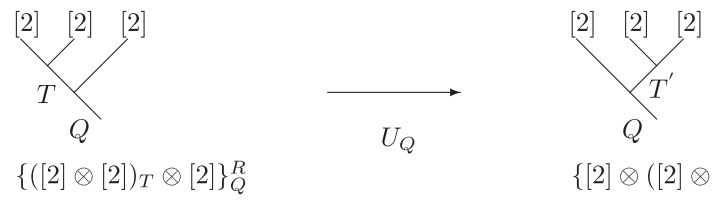

$\left\{[2] \otimes([2] \otimes[2])_{T^{\prime}}\right\}_{Q}^{L}$

Here, $\quad Q \in\{[2,2,2],[6,0],[3,3],[4,1,1], 2[5,1], 2[3,2,1]$, $3[4,2]\}$ and $T, T^{\prime} \in\{[4],[2,2],[3,1]\}$.

For example, the order of matrix $U_{[5,1]}$ is $2 \times 2$ and $U_{[4,2]}$ is $3 \times 3$. The highest weight vector $H_{T}$ for $T \in$ $[2]^{\otimes 2}$ can be determined by applying the raising operator $\Delta T_{1}^{+}$on product $V_{0,0} \otimes V_{0,0}$. Clearly, $H_{[4]}=V_{0,0,0,0}$. We will now elaborate the steps involved for $H_{[3,1]}$ for clarity:

$$
\begin{aligned}
H_{[3,1]} & =\alpha\left\{V_{0,0} \otimes\left(\Delta\left(T_{1}^{+}\right) V_{0,0}\right)\right\}+\beta\left\{\left(\Delta\left(T_{1}^{+}\right) V_{0,0}\right) \otimes V_{0,0}\right\} \\
& =\alpha\left\{V_{0,0,0,1}+q V_{0,0,1,0}\right\}+\beta\left\{V_{0,1,0,0}+q V_{1,0,0,0}\right\} .
\end{aligned}
$$

To find $\alpha$ and $\beta$, one should impose the highest weight vector condition $\Delta\left(T_{1}^{-}\right) H_{[3,1]}=0$, which implies

$\alpha\left\{\left(q^{-3}+q^{-1}\right) V_{0,0,0,0}\right\}+\beta\left\{\left(q+q^{-1}\right) V_{0,0,0,0}\right\}=0$,

giving $\alpha=-q^{+2}, \beta=1$. Hence, the explicit highest vector state is

$$
H_{[3,1]}=-q^{2} V_{0,0,0,1}-q^{3} V_{0,0,1,0}+V_{0,1,0,0}+q V_{1,0,0,0} .
$$

By a similar procedure, one can obtain the highest weight vector for $H_{[2,2]}$ :

$$
\begin{aligned}
H_{[2,2]}= & -q V_{0,0,1,1}-q^{3} V_{0,0,1,1}+V_{0,1,0,1}+q V_{0,1,1,0} \\
& +q V_{1,0,0,1}+q^{2} V_{1,0,1,0}-q^{-1} V_{1,1,0,0}-q V_{1,1,0,0} .
\end{aligned}
$$

Using the result of the highest weight vectors of the $m=2$ strand, we can move to the $m=3$ strand braid and do the explicit calculation of the elements of $U_{Q}$ matrices and construct the inclusive Racah matrices for those representations by following the same steps as above. For the sake of definiteness, we focus on one of the representations, i.e., [5,1], for the $m=3$ strands which has multiplicity of 2 [see (29)]. The representation $[5,1]$ comes from two sectors, the left sector (L), corresponding to [2] $\otimes[4]$ and $[2] \otimes[3,1]$, and the right sector $(R)$, corresponding to $[4] \otimes[2]$ and $[3,1] \otimes[2]$. On representation $Q$, we place a subscript to keep track of the multiplicity and a superscript to denote the left or right sector. Corresponding to the highest weight vectors of the representation [5,1] in each sector is a subscript label 1 and 2 and the unitary matrix for [5,1]. Therefore, to find the $\mathrm{U}$ matrix for the representation $[5,1]$, one has to solve the following equations:

$$
\begin{gathered}
H_{[5,1]_{1}^{R}} \in \underbrace{([4]) \otimes[2]}_{\text {Right sector }}=\underbrace{\alpha([2] \otimes[4])+\beta([2] \otimes[3,1])}_{\text {Left sector }} \\
H_{[5,1]_{2}^{R}} \in \underbrace{([3,1]) \otimes[2]}_{\text {Right sector }}=\underbrace{\gamma([2] \otimes[31])+\delta([2] \otimes[3,1])}_{\text {Left sector }},
\end{gathered}
$$

Now, it remains to present the explicit calculations to determine the unknown parameters $\alpha, \beta, \gamma, \delta$ : Right sector:

$$
\begin{aligned}
{[5,1]_{1}^{R} \in } & {[4] \otimes[2] } \\
H_{[5,1]_{1}^{R}}= & \beta_{R}\left\{V_{0,0,0,0} \otimes\left(\Delta T_{1}^{+} V_{0,0}\right)\right\} \\
& +\alpha_{R}\left\{\left(\Delta T_{1}^{+} V_{0,0,0,0}\right) \otimes V_{0,0}\right\} \\
= & \alpha_{R}\left\{V_{0,0,0,1,0,0}+q V_{0,0,1,0,0,0}+q^{2} V_{0,1,0,0,0,0}\right. \\
& \left.+q^{3} V_{1,0,0,0,0,0}\right\}+\beta_{R}\left\{V_{0,0,0,0,0,1}+q V_{0,0,0,0,1,0}\right\}
\end{aligned}
$$

To find the value of $\alpha_{R}$ and $\beta_{R}$, we apply the highest weight condition:

$$
\begin{aligned}
\Delta\left(T_{1}^{-}\right) H_{[5,1]_{1}^{R}}= & 0 \\
\Rightarrow & \alpha_{R}\left\{\left(q^{-3}+q^{-1}+q+q^{3}\right) V_{0,0,0,0,0,0}\right\} \\
& +\beta_{R}\left\{\left(q^{-3}+q^{-5}\right) V_{0,0,0,0,0,0}\right\}=0 \\
\Rightarrow & \alpha_{R}=-q^{-4} \frac{[2]_{q}}{[4]_{q}}, \quad \beta_{R}=1 .
\end{aligned}
$$

Hence, the final form for the highest weight vector is 


$$
H_{[5,1]_{1}^{R}}=\frac{1}{\mathcal{N}_{\mathcal{R}}}\left\{-q^{-4} \frac{[2]_{q}}{[4]_{q}}\left\{V_{0,0,0,1,0,0}+q V_{0,0,1,0,0,0}+q^{2} V_{0,1,0,0,0,0}+q^{3} V_{1,0,0,0,0,0}\right\}+\left\{V_{0,0,0,0,0,1}+q V_{0,0,0,0,1,0}\right\}\right\},
$$

where the normalization constant is equal to

$$
\mathcal{N}_{\mathcal{R}}=\sqrt{-q^{-1}[6]_{q} \frac{[2]_{q}}{[4]_{q}}}
$$

Left sector:

$$
\begin{aligned}
{[5,1]_{1}^{L} } & \in[2] \otimes[4] \\
H_{[5,1]_{1}^{L}} & =\alpha_{L}\left\{V_{0,0} \otimes\left(\Delta T_{1}^{+} V_{0,0,0,0}\right)\right\}+\beta_{L}\left\{\left(\Delta T_{1}^{+} V_{0,0}\right) \otimes V_{0,0,0,0}\right\} \\
& =\alpha_{L}\left\{V_{0,0,0,0,0,1}+q V_{0,0,0,0,1,0}+q^{2} V_{0,0,0,1,0,0}+q^{3} V_{0,0,1,0,0,0}\right\}+\beta_{L}\left\{V_{0,1,0,0,0,0}+q V_{1,0,0,0,0,0}\right\} \\
\Delta\left(T_{1}^{-}\right) H_{[5,1]_{1}^{L}} & =0 \\
& \Rightarrow \alpha_{L}\left\{\left(q^{-5}+q^{-3}+q^{-1}+q\right) V_{0,0,0,0,0,0}\right\}+\beta_{L}\left\{\left(q^{-1}+q\right) V_{0,0,0,0,0,0}\right\}=0 \\
& \Rightarrow \alpha_{L}=-q^{2} \frac{[2]_{q}}{[4]_{q}}, \quad \beta_{L}=1 .
\end{aligned}
$$

Hence, the final form for the highest weight vector is

$$
H_{[5,1]_{1}^{L}}=\frac{1}{\mathcal{N}_{\mathcal{L}}}\left\{-q^{2} \frac{[2]_{q}}{[4]_{q}}\left\{V_{0,0,0,0,0,1}+q V_{0,0,0,0,1,0}+q^{2} V_{0,0,0,1,0,0}+q^{3} V_{0,0,1,0,0,0}\right\}+\left\{V_{0,1,0,0,0,0}+q V_{1,0,0,0,0,0}\right\}\right\},
$$

where the normalization constant is equal to

$$
\mathcal{N}_{\mathcal{L}}=\sqrt{-q^{-3}[6]_{q} \frac{[2]_{q}}{[4]_{q}}}
$$

Similarly, one can construct the highest weight vectors $H_{[5,1]_{2}^{R}}$ and $H_{[5,1]_{2}^{L}}$ which come from representation [3,1]. Hence $\alpha, \beta, \gamma$, and $\delta$ for the matrix $U_{[5,1]}$ are equal to:

\begin{tabular}{lcc}
\hline$U_{[5,1]}$ & $H_{[5,1]_{1}^{L}}$ & $H_{[5,1]_{2}^{L}}$ \\
\hline$H_{[5,1]_{1}^{R}}$ & $\alpha=\frac{q^{2}}{\left(1+q^{4}\right)}$ & $\beta=\sqrt{\frac{1+q^{4}+q^{8}}{\left(1+q^{4}\right)^{2}}}$ \\
$H_{[5,1]_{2}^{R}}$ & $\gamma=-\sqrt{\frac{1+q^{4}+q^{8}}{\left(1+q^{4}\right)^{2}}}$ & $\delta=\frac{q^{2}}{\left(1+q^{4}\right)}$ \\
\hline
\end{tabular}

Similarly, one can obtain the $U$ matrices for all the representations in $m=3$ case. Interestingly, the magnitude of all the elements coincide with the result obtained from the eigenvalue hypothesis in Eqs. (16) and (22). In the following section, we aim to compare the inclusive Racah matrices with the matrices given in Sec. III for the $m=4$ strands.

\section{B. Evaluation of the $U$ and $W_{1}$ matrix for $m=4$}

The detailed procedure which we discussed for determining the $U$ matrices for three-strand braids can be extended to four-strand braids. Here, we need to consider $Q \vdash[2]^{\otimes 4}$. The possible representations $Q$ with their multiplicities are tabulated below:

\begin{tabular}{lcc}
\hline \multicolumn{1}{c}{$m=4$ strand braid } & $\begin{array}{c}\text { Matrix } \\
\text { size }\end{array}$ & $\begin{array}{c}\# \text { of } \\
\text { matrices }\end{array}$ \\
$\mathbf{Q}$ & 1 & 3 \\
{$[2,2,2,2],[5,1,1,1],[8,0]$} & 2 & 1 \\
{$[3,3,1,1]$} & 3 & 6 \\
{$[3,2,2,1],[3,3,2],[4,2,1,1],[4,4],[6,1,1],[7,1]$} & 6 & 3 \\
{$[4,2,2],[5,3],[6,2]$} & 7 & 1 \\
{$[4,3,1]$} & 8 & 1 \\
{$[5,2,1]$} & & \\
\hline
\end{tabular}

From (9), we observe that the matrix $U W_{1} U$ relates the right sector highest weight vector with the corresponding left sector highest weight vector. We present the calculations for representation [6,2] (see Fig. 1). Let us emphasize that the subsector states involving three-strands belong to different sectors. In order to obtain $U$ and $W_{1}$, we need to determine the matrix $U W_{1}$ as well. In fact, the matrix $U W_{1}$ relates the two highest weight vectors of different sectors, but both their subsectors are either left or right sector. We have highlighted them for representation [6,2] in Fig. 1.

It is clear from Fig. 1 that there are six independent paths to obtain representation [6,2]. Therefore, both $U W_{1} U$ and 


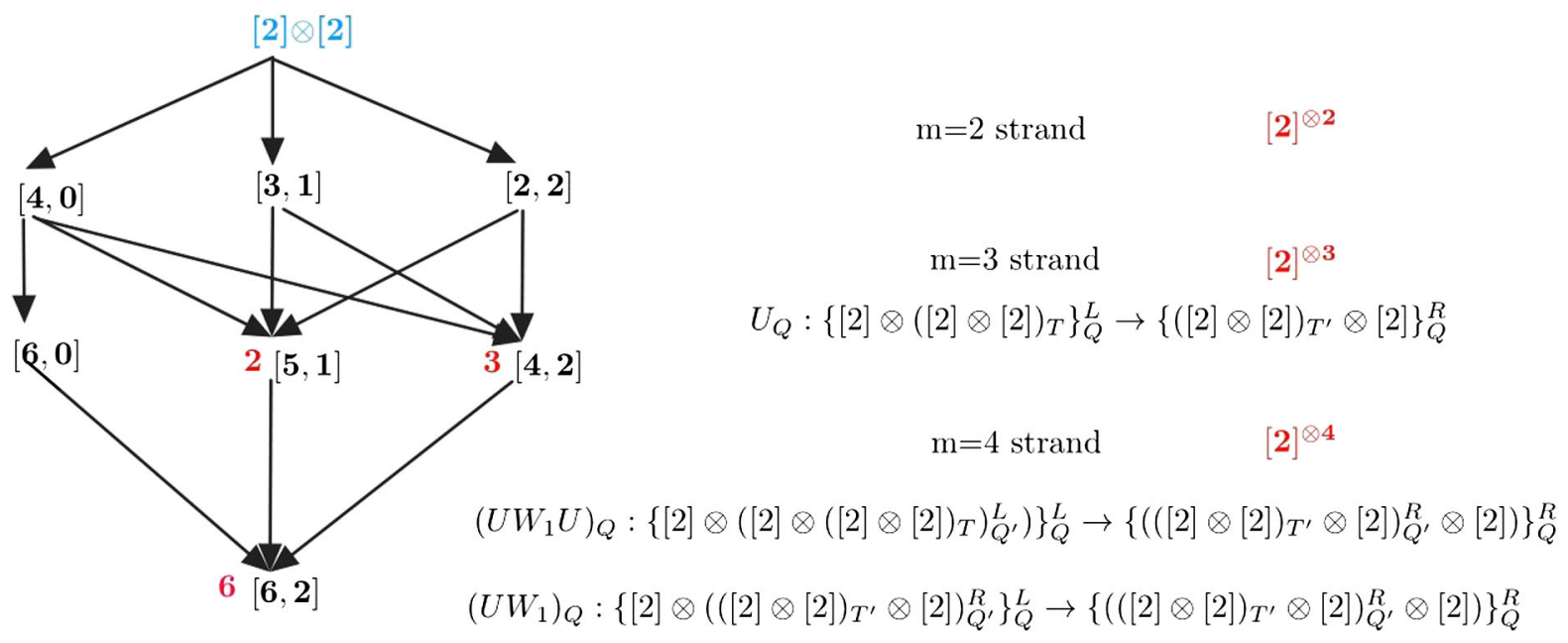

FIG. 1. Steps for $U W_{1} U$ unitary matrices computation for $m=4$ strands.

$U W_{1}$ matrices will be of the size $6 \times 6$. Following the highest weight method procedure, one can work out the six right sector highest weight vectors $[6,2]_{i}^{R}$ and similarly the six left sector highest weight vectors $[6,2]_{j}^{L}$, whose subsectors are different. Hence, one can determine the 36 matrix elements of $U W_{1} U$ by taking inner product of the left and right sector states.

One could again work out six highest weight states for left and right sectors but with both subsectors being the same. The inner product of such states will give the elements of the $U W_{1}$ matrix. We present the $U$ and $W_{1}$ whose matrix sizes are less than $6 \times 6$ in the following section. Other matrices of the sizes $n \times n$ for $n \geq 6$ are presented in Appendix A.

\section{EXAMPLES}

For clarity, we will first review and give the matrices for the simplest case when the strands carry fundamental representation $R=[1]$. Then, the case $R=[2]$ will be presented.

\section{A. Representation [1]}

In this case, $[1]^{\otimes 4}=[4]+3[3,1]+2[2,2]+3[2,1,1]+$ $[1,1,1,1]$. Thus, there are two matrices of size $1 \times 1$, one matrix of size $2 \times 2$, and two matrices of size $3 \times 3$. The eigenvalues of $\mathcal{R}$ matrices are in this case $\lambda_{1}=q$, $\lambda_{2}=-q^{-1}$. The only nontrivial case here is matrices of size $3 \times 3$. The $\mathcal{R}$ matrices are equal to

$$
\mathcal{R}^{[3,1]}=\left(\begin{array}{ccc}
q & & \\
& q & \\
& & -q^{-1}
\end{array}\right), \quad \mathcal{R}^{[2,1,1]}=\left(\begin{array}{ccc}
q & & \\
& -q^{-1} & \\
& & -q^{-1}
\end{array}\right) .
$$

Then, the $U$ matrices are equal to

$$
U^{[3,1]}=\left(\begin{array}{ccc}
1 & & \\
& \frac{1}{[2]} & \frac{\sqrt{[3]}}{[2]} \\
& \frac{\sqrt{[3]}}{[2]} & -\frac{1}{[2]}
\end{array}\right), \quad U^{[2,1,1]}=\left(\begin{array}{ccc}
\frac{1}{[2]} & \frac{\sqrt{[3]}}{[2]} & \\
\frac{\sqrt{[3]}}{[2]} & -\frac{1}{[2]} & \\
& & 1
\end{array}\right),
$$

and the $W_{1}$ matrices are equal to 


$$
W_{1}^{[3,1]}=\left(\begin{array}{ccc}
\frac{1}{[3]} & \frac{\sqrt{[2][4]}}{[3]} & \\
\frac{\sqrt{[2][4]}}{[3]} & -\frac{1}{[3]} & \\
& & 1
\end{array}\right), \quad W_{1}^{[2,1,1]}=\left(\begin{array}{ccc}
1 & & \\
& \frac{1}{[3]} & \frac{\sqrt{[2][4]}}{[3]} \\
\frac{\sqrt{[2][4]}}{[3]} & -\frac{1}{[3]}
\end{array}\right),
$$

which is in full accordance with formulas (22) and (23) from Sec. III B.

\section{B. Representation [2]}

The representations $Q$ for the four-strand braid carrying representation $R=$ [2] are

$$
\begin{aligned}
{[2]^{\otimes 4}=} & {[8]+3[7,1]+6[6,2]+3[6,1,1]+6[5,3]+8[5,2,1]+[5,1,1,1]+3[4,4]+7[4,3,1] } \\
& +6[4,2,2]+3[4,2,1,1]+3[3,3,2]+2[3,3,1,1]+3[3,2,2,1]+[2,2,2,2] .
\end{aligned}
$$

Thus, there are five $3 \times 3$ matrices, three $6 \times 6$ matrices, one $7 \times 7$, and one $8 \times 8$ matrices. The eigenvalues in this case are $\lambda_{1}=q^{6}, \lambda_{2}=-q^{2}$, and $\lambda_{3}=1$. For the size $3 \times 3$, the matrices are:

$$
\begin{aligned}
& \mathcal{R}^{[7,1]}=\left(\begin{array}{ccc}
q^{6} & & \\
& q^{6} & \\
& & -q^{2}
\end{array}\right), \quad \mathcal{R}^{[6,1,1]}=\left(\begin{array}{ccc}
q^{6} & & \\
& -q^{2} & \\
& & -q^{2}
\end{array}\right), \quad \mathcal{R}^{[4,4]}=\left(\begin{array}{lll}
q^{6} & & \\
& -q^{2} & \\
& & 1
\end{array}\right), \\
& \mathcal{R}^{[4,2,1,1]}=\mathcal{R}^{[3,3,2]}=\left(\begin{array}{ccc}
-q^{2} & & \\
& -q^{2} & \\
& & 1
\end{array}\right), \quad \mathcal{R}^{[3,2,2,1]}=\left(\begin{array}{ccc}
-q^{2} & & \\
& 1 & \\
& & 1
\end{array}\right), \quad \mathcal{R}^{[3,3,1,1]}=\left(\begin{array}{ll}
-q^{2} & \\
& 1
\end{array}\right),
\end{aligned}
$$

Then, the $U$ matrices are equal to

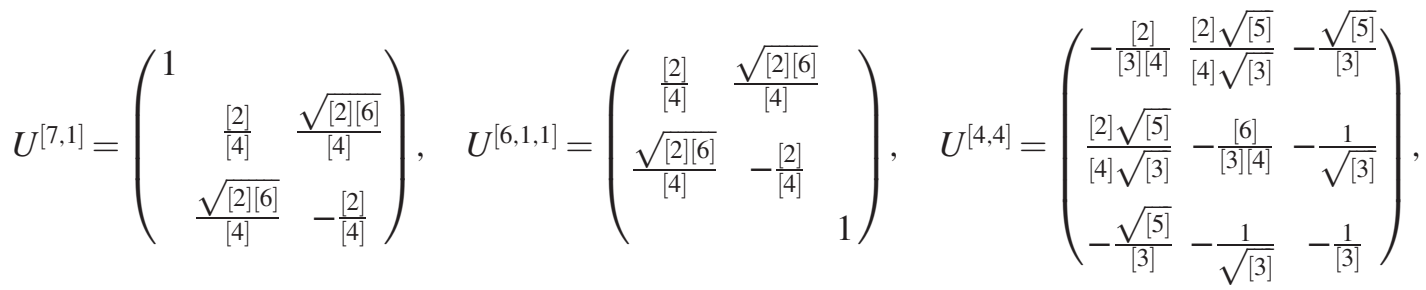

$$
\begin{aligned}
& U^{[3,2,2,1]}=\left(\begin{array}{ccc}
-\frac{1}{[2]} & -\frac{\sqrt{[3]}}{[2]} & \\
-\frac{\sqrt{[3]}}{[2]} & \frac{1}{[2]} & \\
& & 1
\end{array}\right), \quad U^{[4,2,1,1]}=U^{[3,3,2]}=\left(\begin{array}{ccc}
1 & & \\
& \frac{1}{[2]} & \frac{\sqrt{[3]}}{[2]} \\
& \sqrt{[3]} & -\frac{1}{[2]}
\end{array}\right), \quad U^{[3,3,1,1]}=\left(\begin{array}{cc}
\frac{1}{[2]} & \frac{\sqrt{[3]}}{[2]} \\
\frac{\sqrt{[3]}}{[2]} & -\frac{1}{[2]}
\end{array}\right),
\end{aligned}
$$

while the corresponding $W_{1}$ matrices are equal to

$$
\begin{aligned}
& W_{1}^{[7,1]}=\left(\begin{array}{ccc}
\frac{[2]}{[6]} & \frac{\sqrt{[4][8]}}{[6]} & \\
\frac{\sqrt{[4][8]}}{[6]} & -\frac{[2]}{[6]} & \\
\hline[6] & & 1
\end{array}\right), \quad W_{1}^{[6,1,1]}=\left(\begin{array}{ccc}
1 & \\
-\frac{[2]}{[6]} & -\frac{\sqrt{[4][8]}}{[6]} \\
-\frac{\sqrt{[4][8]}}{[6]} & \frac{[2]}{[6]}
\end{array}\right), \quad W_{1}^{[4,4]}=\left(\begin{array}{ccc}
1 & & \\
& 1 & \\
& & 1
\end{array}\right),
\end{aligned}
$$

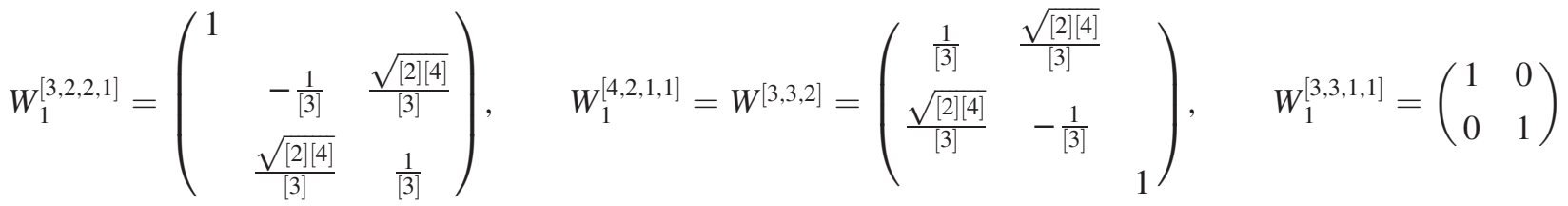

which is in a full accordance with formulas (22) and (23) from Sec. III B.

Matrices having larger sizes are provided in Appendix A. 


\section{CONCLUSION}

In the present paper, we managed to present the generalization of the eigenvalue hypothesis suggested in [28] for three-strands to a multistrand case. We claim that, while the three-strand eigenvalue hypothesis comes from the YangBaxter equation, its generalization is based on solving the commutativity relations of non-neighboring $\mathcal{R}$ matrices. In particular, in the case of the four-strand braid, there is one commutativity relation. Solving it provided us with the eigenvalue answers for the four-strand Racah matrices of sizes up to $6 \times 6$. Calculating matrices of the larger sizes encountered some computation difficulties, and, thus, they have not been found yet. However, all the eigenvalue formulas for the Racah matrices up to size $6 \times 6$ have been checked by calculating the matrices for representations [1] and [2] using the highest weight method. The problem of finding the answers for matrices of larger sizes still remains.

Thus, the eigenvalue hypothesis for the particular example of the four-strand braids as well as the highest weight method allowed us to provide all the Racah matrices for the four-strand braids carrying representation [2]. This enabled us to obtain the HOMFLY-PT polynomials for knots from four-strand braids. Moreover, one can check the obtained results for the Racah matrices comparing known HOMFLYPT polynomials in representation [2] for various knots with those evaluated using the results of the present paper. We confirmed these results by comparing with correct answers for the HOMFLY-PT polynomials for: torus knots [38], twist knots $6_{1}$ and $7_{2}[9,39]$, and many arborescent knots that have four-strand braid representation $[7,15]$.
In Appendix B, we list a few nontrivial examples of HOMFLY-PT polynomials in the first symmetric representation that are so far unknown: knots in accordance with the Rolfsen table [40] which are nonarborescent and are described by four-strand braids: $9_{34}, 9_{40}, 9_{47}, 9_{49} 10_{102}$, $10_{103}, 10_{108}, 10_{111}, 10_{113}, 10_{114}, 10_{117}, 10_{119}, 10_{121}, 10_{122}$, $10_{156}, 10_{158}, 10_{160}, 10_{162}-10_{165}$.

The eigenvalue hypothesis in the multistrand case was already related in [32] to the well-established property [14] $A l_{R}(q)=A l_{[1]}\left(q^{|R|}\right)$ of the Alexander polynomials colored by the single-hook diagrams, which provided an indirect support for it. In this text, we found direct evidence in favor of this hypothesis and concrete formulas for its realization in the case of $\mathcal{R}$ matrices of small sizes. Extension to matrices of arbitrary size for any number of strands including three remains a challenging problem.

\section{ACKNOWLEDGMENTS}

Our work was partly supported by a grant from the Foundation for the Advancement of Theoretical Physics "BASIS" (Alexei Morozov and Alexey Sleptsov), by RFBR Grants No. 16-01-00291 (Andrei Mironov), No. 16-0201021 (Alexei Morozov), and No. 17-01-00585 (Andrey Morozov), by joint Grants No. 17-51-50051-YaF, 15-5152031-NSC-a, No. 16-51-53034-GFEN, No. 16-51-45029IND-a (Andrei Mironov, Alexei Morozov and Andery Morozov), and by Grant No. 16-31-60082-mol-a-dk (Alexey Sleptsov). Pichai Ramadevi, Vivek Kumar Singh, and Saswati Dhara acknowledge a DST-RFBR Grant (INT/ RUS/RFBR/P-231) for support. Saswati Dhara would like to thank CSIR for a research fellowship.

\section{APPENDIX A: REPRESENTATION [2] MATRICES}

In this appendix, all the Racah matrices of sizes larger than $3 \times 3$ for the four-strand braid in the first symmetric representation are provided. The matrices of the size $6 \times 6$ are in full accordance with formulas (24) and (25) from Sec. III B.

The diagonal $\mathcal{R}$ matrices look like

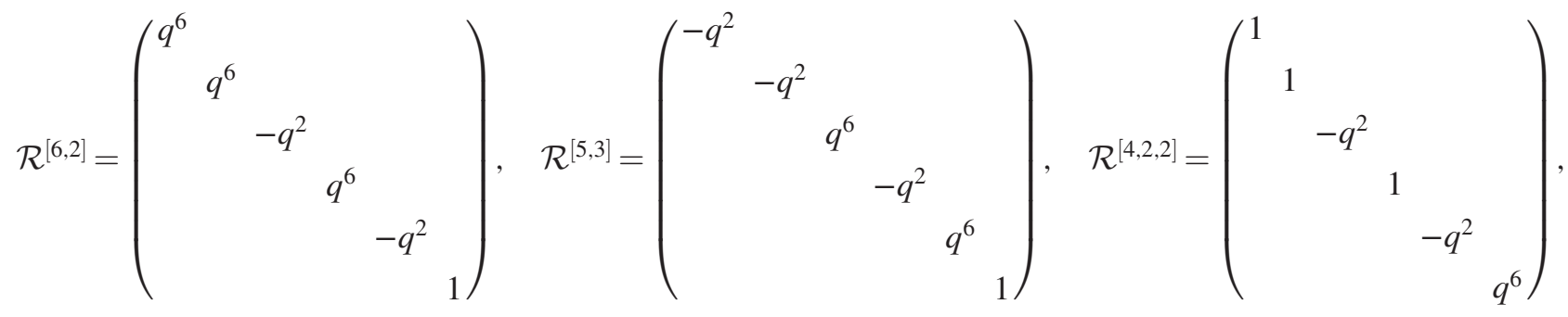

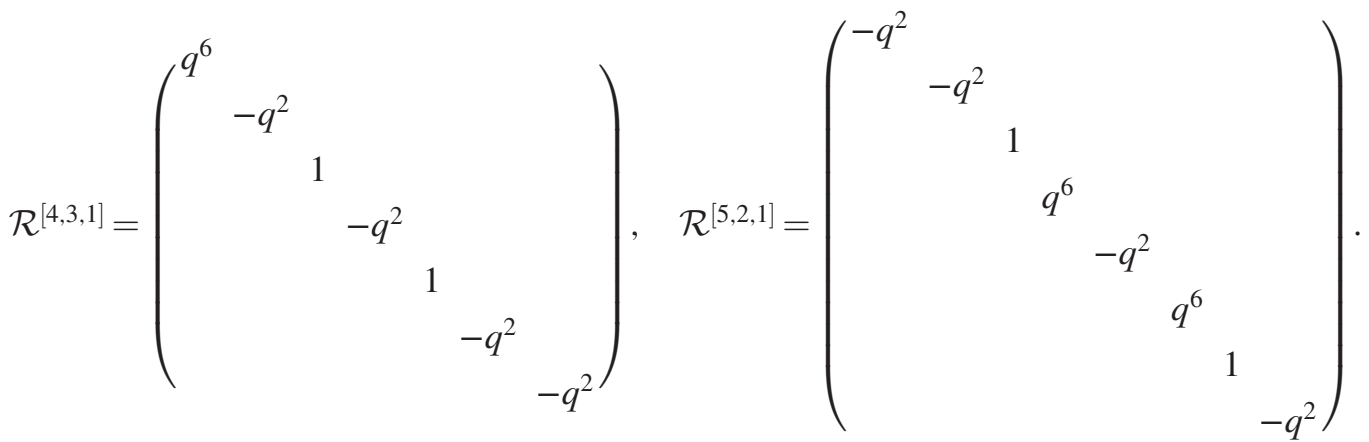


The $U$ matrices are equal to

$$
\begin{aligned}
& U^{[6,2]}=\left(\begin{array}{cccccc}
1 & 0 & 0 & 0 & 0 & 0 \\
0 & -\frac{[2]}{[4]} & \frac{\sqrt{[2][6]}}{[4]} & 0 & 0 & 0 \\
0 & -\frac{\sqrt{[2][6]}}{[4]} & -\frac{[2]}{[4]} & 0 & 0 & 0 \\
0 & 0 & 0 & \frac{[2]}{[3][4]} & -\frac{[2]}{[4]} \sqrt{[5]} & \frac{\sqrt{[5]}}{[3]} \\
0 & 0 & 0 & \frac{[2]}{[4]} \sqrt{\frac{[5]}{[3]}} & -\frac{[6]}{[3][4]} & -\frac{1}{\sqrt{[3]}} \\
0 & 0 & 0 & \frac{\sqrt{[5]}}{[3]} & \frac{1}{\sqrt{[3]}} & \frac{1}{[3]}
\end{array}\right), \\
& U^{[4,2,2]}=\left(\begin{array}{cccccc}
1 & 0 & 0 & 0 & 0 & 0 \\
0 & -\frac{1}{[2]} & \frac{\sqrt{[3]}}{[2]} & 0 & 0 & 0 \\
0 & -\frac{\sqrt{[3]}}{[2]} & -\frac{1}{[2]} & 0 & 0 & 0 \\
0 & 0 & 0 & \frac{1}{[3]} & -\frac{1}{\sqrt{[3]}} & -\frac{\sqrt{[5]}}{[3]} \\
0 & 0 & 0 & \frac{1}{\sqrt{[3]}} & -\frac{[6]}{[3][4]} & \frac{[2]}{[4]} \sqrt{[5]} \\
0 & 0 & 0 & -\frac{\sqrt{[5]}}{[3]} & -\frac{[2]}{[4]} \sqrt{\frac{[5]}{[3]}} & \frac{[2]}{[3][4]}
\end{array}\right), \\
& U^{[5,3]}=\left(\begin{array}{cccccc}
1 & 0 & 0 & 0 & 0 & 0 \\
0 & -\frac{[2]}{[4]} & -\frac{\sqrt{[2][6]}}{[4]} & 0 & 0 & 0 \\
0 & \frac{\sqrt{[2][6]}}{[4]} & -\frac{[2]}{[4]} & 0 & 0 & 0 \\
0 & 0 & 0 & \frac{-[6]}{[4][3]} & \frac{[2]}{[4]} \sqrt{[5]} & -\frac{1}{\sqrt{[3]}} \\
0 & 0 & 0 & -\frac{[2]}{[4]} \sqrt{\frac{[5]}{[3]}} & \frac{[2]}{[3][4]} & \frac{\sqrt{[5]}}{[3]} \\
0 & 0 & 0 & \frac{1}{\sqrt{[3]}} & \frac{\sqrt{[5]}}{[3]} & \frac{1}{[3]}
\end{array}\right), \\
& U^{[4,3,1]}=\left(\begin{array}{ccccccc}
\frac{[2]}{[3][4]} & \frac{[2][5]}{[4] \sqrt{[3][5]}} & \frac{\sqrt{[5]}}{[3]} & 0 & 0 & 0 & 0 \\
\frac{[2][5]}{[4] \sqrt{[3][5]}} & \frac{[6]}{[3][4]} & -\frac{1}{\sqrt{[3]}} & 0 & 0 & 0 & 0 \\
\frac{\sqrt{[5]}}{[3]} & -\frac{1}{\sqrt{[3]}} & \frac{1}{[3]} & 0 & 0 & 0 & 0 \\
0 & 0 & 0 & \frac{1}{[2]} & \frac{\sqrt{[3]}}{[2]} & 0 & 0 \\
0 & 0 & 0 & \frac{\sqrt{[3]}}{[2]} & -\frac{1}{[2]} & 0 & 0 \\
0 & 0 & 0 & 0 & 0 & 1 & 0 \\
0 & 0 & 0 & 0 & 0 & 0 & 1
\end{array}\right),
\end{aligned}
$$




$$
U^{[5,2,1]}=\left(\begin{array}{cccccccc}
1 & 0 & 0 & 0 & 0 & 0 & 0 & 0 \\
0 & \frac{1}{[2]} & \frac{\sqrt{[3]}}{[2]} & 0 & 0 & 0 & 0 & 0 \\
0 & \frac{\sqrt{[3]}}{[2]} & \frac{-1}{[2]} & 0 & 0 & 0 & 0 & 0 \\
0 & 0 & 0 & \frac{[2]}{[4]} & \frac{\sqrt{[2][6]}}{[4]} & 0 & 0 & 0 \\
0 & 0 & 0 & \frac{\sqrt{[2][6]}}{[4]} & -\frac{[2]}{[4]} & 0 & 0 & 0 \\
0 & 0 & 0 & 0 & 0 & \frac{[2]}{[3][4]} & \frac{\sqrt{[5]}}{[3]} & -\frac{[2][5]}{[4] \sqrt{[3][5]}} \\
0 & 0 & 0 & 0 & 0 & \frac{\sqrt{[5]}}{[3]} & \frac{1}{[3]} & \frac{1}{\sqrt{[3]}} \\
0 & 0 & 0 & 0 & 0 & \frac{[2][5]}{[4] \sqrt{[3][5]}} & -\frac{1}{\sqrt{[3]}} & -\frac{[6]}{[3][4]}
\end{array}\right) .
$$

The $W_{1}$ matrices are equal to

$$
W_{1}^{[6,2]}=\left(\begin{array}{cccccc}
\frac{[2]}{[5][6]} & \frac{[2] \sqrt{\frac{[7]}{[5}}}{[6]} & 0 & \frac{\sqrt{[3][7]}}{[5]} & 0 & 0 \\
\frac{[2] \sqrt{\frac{77]}{[5]}}}{[6]} & 1-\frac{[2]^{2}}{[4][6]} & 0 & -\frac{[2]}{[4]} \sqrt{\frac{[3]}{[5]}} & 0 & 0 \\
0 & 0 & -\frac{[2]}{[4]} & 0 & -\frac{\sqrt{[2][6]}}{[4]} & 0 \\
\frac{\sqrt{[3][7]}}{[5]} & -\frac{[2]}{[4]} \sqrt{\frac{[3]}{[5]}} & 0 & \frac{[2]}{[4][5]} & 0 & 0 \\
0 & 0 & -\frac{\sqrt{[2][6]}}{[4]} & 0 & \frac{[2]}{[4]} & 0 \\
0 & 0 & 0 & 0 & 0 & 1
\end{array}\right),
$$




$$
\begin{aligned}
& W_{1}^{[5,3]}=\left(\begin{array}{cccccc}
\frac{1}{[3]} & \frac{\sqrt{[5]}}{[3]} & 0 & -\frac{1}{\sqrt{[3]}} & 0 & 0 \\
\frac{\sqrt{[5]}}{[3]} & \frac{[2]}{[4][3]} & 0 & \frac{[2]}{[4]} \sqrt{[5]} & 0 & 0 \\
0 & 0 & -\frac{[2]}{[4]} & 0 & -\frac{\sqrt{[2][6]}}{[4]} & 0 \\
-\frac{1}{\sqrt{[3]}} & \frac{[2]}{[4]} \sqrt{\frac{[5]}{[3]}} & 0 & \frac{[6]}{[4][3]} & 0 & 0 \\
0 & 0 & -\frac{\sqrt{[2][6]}}{[4]} & 0 & \frac{[2]}{[4]} & 0 \\
0 & 0 & 0 & 0 & 0 & -1
\end{array}\right), \\
& W_{1}^{[5,2,1]}=\left(\begin{array}{cccccccc}
\frac{[8]}{[4][6]} & -\frac{\sqrt{[2]}}{\sqrt{[3][6]}} & 0 & 0 & -\frac{\sqrt{[7]}}{[6]} & 0 & 0 & \frac{\sqrt{[7]}}{\sqrt{[2][6]}} \\
\frac{\sqrt{[2]}}{\sqrt{[3][6]}} & -\frac{[2]}{[3][5]} & 0 & 0 & \frac{\sqrt{[2][7]}}{\sqrt{[3][6]}} & 0 & 0 & \frac{[7]}{[5] \sqrt{[3][7]}} \\
0 & 0 & \frac{[2]}{[5]} & 0 & 0 & 0 & -\frac{\sqrt{[3][7]}}{[5]} & 0 \\
0 & 0 & 0 & \frac{1}{[4]} & 0 & -\frac{\sqrt{[3][5]}}{[4]} & 0 & 0 \\
\frac{\sqrt{[7]}}{[6]} & \frac{\sqrt{[2][7]}}{\sqrt{[3][6]}} & 0 & 0 & -\frac{[2]}{[4][6]} & 0 & 0 & \frac{\sqrt{[2]}}{[4] \sqrt{[6]}} \\
0 & 0 & 0 & -\frac{\sqrt{[3][5]}}{[4]} & 0 & -\frac{1}{[4]} & 0 & 0 \\
0 & 0 & -\frac{\sqrt{[3][7]}}{[5]} & 0 & 0 & 0 & -\frac{[2]}{[5]} & 0 \\
\frac{\sqrt{[7]}}{\sqrt{[2][6]}} & -\frac{[7]}{[5] \sqrt{[3][7]}} & 0 & 0 & -\frac{\sqrt{[2]}}{[4] \sqrt{[6]}} & 0 & 0 & -\frac{[1]+[3]+[7]}{[4][5]}
\end{array}\right), \\
& W_{1}^{[4,3,1]}=\left(\begin{array}{ccccccc}
1 & 0 & 0 & 0 & 0 & 0 & 0 \\
0 & \frac{[6]}{[3][2][5]} & 0 & \frac{[4] \sqrt{[4][6]}}{[2][3][5]} & 0 & \frac{[4]}{[2] \sqrt{[3][5]}} & \frac{[4]}{[2] \sqrt{[3][5]}} \\
0 & 0 & -\frac{1}{[5]} & 0 & \frac{\sqrt{[4][6]}}{[5]} & 0 & 0 \\
0 & \frac{[4] \sqrt{[4][6]}}{[2][3][5]} & 0 & \frac{[4]+[2][5]}{[2][3][5]} & 0 & -\frac{\sqrt{\frac{[4][6]}{[3][5]}}}{[3]} & -\frac{\sqrt{\frac{[4][6]}{[3][5]}}}{[3]} \\
0 & 0 & \frac{\sqrt{[4][6]}}{[5]} & 0 & \frac{1}{[5]} & 0 & 0 \\
0 & \frac{[4]}{[2] \sqrt{[3][5]}} & 0 & -\frac{\sqrt{\frac{44[6]}{[3] 5]}}}{[3]} & 0 & -\frac{1}{[3]} & \frac{[4]}{[2][3]} \\
0 & \frac{[4]}{[2] \sqrt{[3][5]}} & 0 & -\frac{\sqrt{[4][6]}}{[3]} & 0 & \frac{[4]}{[2][3]} & -\frac{1}{[3]}
\end{array}\right) .
\end{aligned}
$$

\section{APPENDIX B: POLYNOMIAL EXAMPLES}

In this appendix, four-strand HOMFLY polynomials in representation [2] are listed. We calculated these polynomials using the matrices from Appendix A and present them in the matrix form suggested in [4]. The matrix describes the coefficients of a polynomial in $A^{2}$ and $q^{2}$ as 


$$
q^{10} A^{16}\left(\begin{array}{ll}
3 & 4 \\
1 & 2
\end{array}\right)=q^{10} A^{16}+2 q^{12} A^{16}+3 q^{10} A^{18}+4 q^{12} A^{18}
$$

HOMFLY polynomials for knots $6_{1}$ and $7_{2}$ are already known since those are arborescent knots, and the answers calculated using four-strand representations coincide with those. All other polynomials presented here were unknown before since those are nonarborescent knots. Their limit for $A=q^{2}$, colored Jones polynomials, are known and are correct. Other limits (Alexander $A=1$ and special $q=1$ polynomials) are known because their dependence on the representation is quite simple $[8,41]$ :

$\mathcal{A}_{V}(q)=H_{V}(A=1, q), \quad \mathcal{A}_{V}(q)=P_{[1]}\left(q^{|V|}\right), \quad V$-hook diagram $\quad \sigma_{V}(A)=H_{V}(A, q=1), \quad \sigma_{V}(A)=\left(\sigma_{[1]}(A)\right)^{|V|}$.

These limits of the polynomials below are also correct.

$$
H_{[2]}^{6_{1}}=\frac{1}{q^{10} A^{8}}\left(\begin{array}{ccccccccc}
0 & 0 & 0 & 0 & 0 & 0 & 0 & 1 & 0 \\
0 & 0 & 0 & 0 & -1 & 0 & 2 & 0 & -1 \\
0 & 0 & 1 & -2 & -2 & 4 & 0 & -3 & 0 \\
0 & 1 & -1 & -2 & 3 & 2 & -2 & 0 & 1 \\
1 & -1 & -1 & 2 & 1 & -1 & 0 & 0 & 0 \\
-1 & -1 & 1 & 0 & -1 & 0 & 0 & 0 & 0 \\
0 & 1 & 0 & 0 & 0 & 0 & 0 & 0 & 0
\end{array}\right)
$$




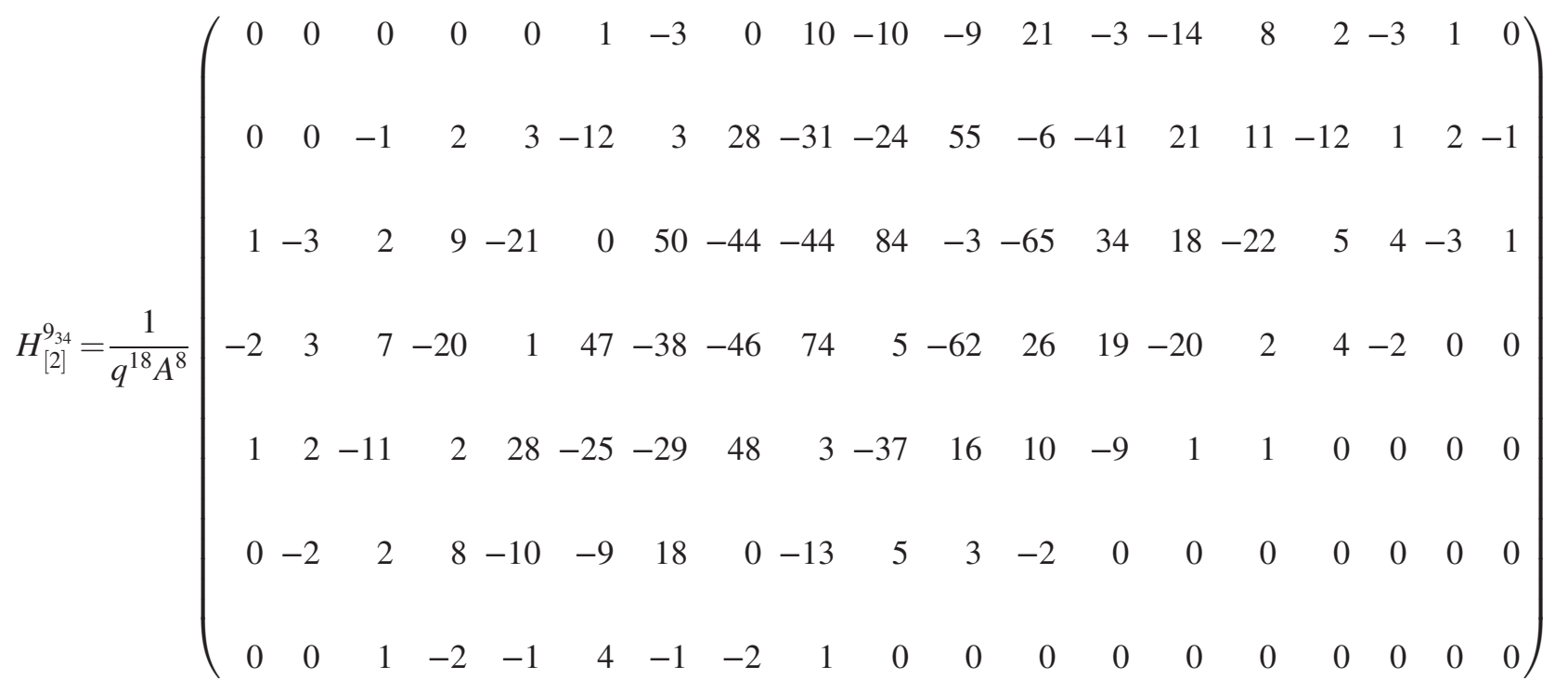

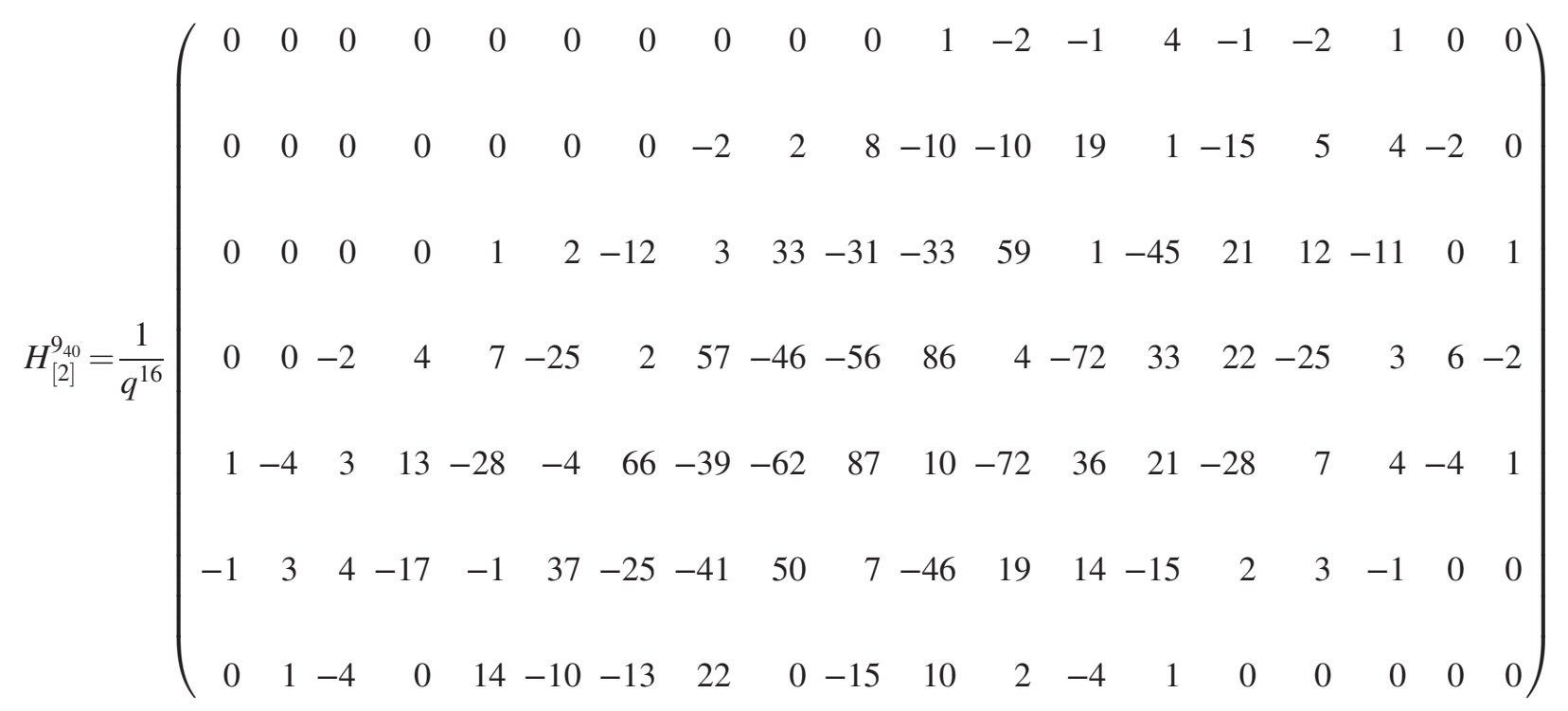

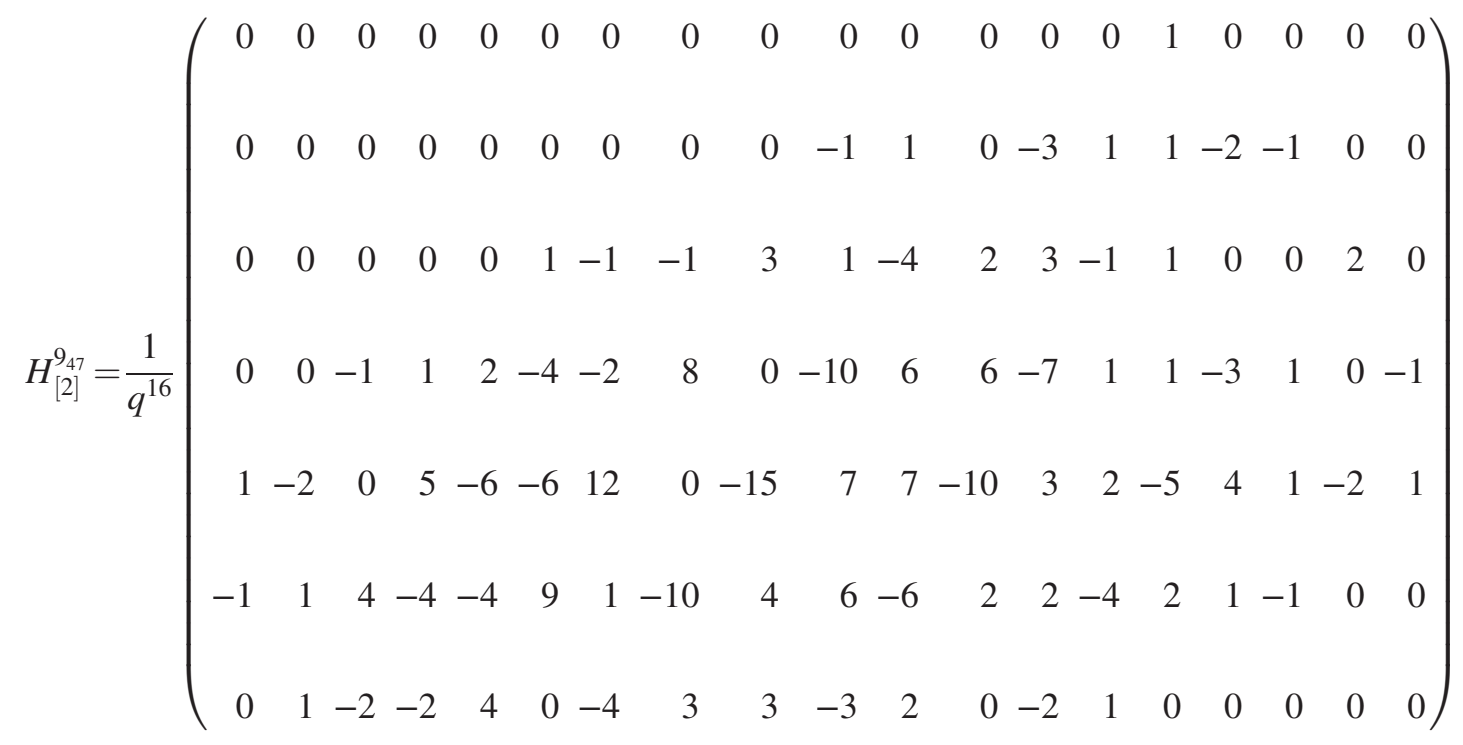




$$
H_{[2]}^{9_{49}}=\frac{A^{8}}{q^{8}}\left(\begin{array}{ccccccccccccccc}
0 & 0 & 0 & 0 & 0 & 0 & 0 & 0 & 0 & 0 & -1 & 0 & 1 & 0 & 0 \\
0 & 0 & 0 & 0 & 0 & 0 & 1 & 4 & -1 & -2 & 5 & 2 & -2 & 2 & 0 \\
0 & 0 & 0 & -1 & -4 & 0 & 4 & -9 & -8 & 7 & -3 & -8 & 2 & -1 & -3 \\
0 & 1 & 1 & -5 & 3 & 12 & -7 & -7 & 16 & 0 & -7 & 8 & 1 & -3 & 3 \\
2 & 1 & -6 & 1 & 10 & -7 & -9 & 11 & 0 & -8 & 6 & 0 & -3 & 2 & 0 \\
1 & -2 & -1 & 5 & -2 & -5 & 5 & 1 & -4 & 3 & 0 & -2 & 1 & 0 & 0
\end{array}\right)
$$

$$
H_{[2]}^{10_{102}}=\frac{1}{q^{18} A^{4}}\left(\begin{array}{ccccccccccccccccccccc}
0 & 0 & 0 & 0 & 0 & 0 & 0 & 1 & -2 & 0 & 5 & -6 & -2 & 10 & -5 & -5 & 6 & 0 & -2 & 1 & 0 \\
0 & 0 & 0 & 0 & -1 & 2 & 1 & -8 & 7 & 8 & -21 & 6 & 22 & -20 & -9 & 18 & -2 & -8 & 3 & 1 & -1 \\
0 & 0 & 0 & -2 & 4 & 2 & -14 & 15 & 12 & -39 & 16 & 39 & -41 & -13 & 37 & -9 & -15 & 10 & 1 & -3 & 1 \\
1 & -1 & -3 & 8 & -1 & -19 & 25 & 12 & -54 & 24 & 50 & -53 & -14 & 44 & -11 & -16 & 11 & 1 & -3 & 1 & 0 \\
0 & -2 & 4 & 0 & -15 & 18 & 15 & -48 & 13 & 51 & -43 & -19 & 38 & -7 & -15 & 9 & 1 & -3 & 1 & 0 & 0 \\
-1 & 2 & 1 & -8 & 8 & 10 & -24 & 3 & 27 & -18 & -12 & 17 & -1 & -7 & 3 & 1 & -1 & 0 & 0 & 0 & 0 \\
0 & 1 & -2 & 0 & 5 & -5 & -3 & 9 & -3 & -5 & 5 & 0 & -2 & 1 & 0 & 0 & 0 & 0 & 0 & 0 & 0
\end{array}\right)
$$

$$
H_{[2]}^{10_{103}}=\frac{1}{q^{24} A^{12}}\left(\begin{array}{cccccccccccccccccccccc}
0 & 0 & 0 & 0 & 0 & 0 & 0 & 1 & -2 & 1 & 4 & -6 & 0 & 8 & -6 & -3 & 6 & -1 & -2 & 1 & 0 \\
0 & 0 & 0 & 0 & -1 & 1 & 2 & -8 & 3 & 12 & -20 & -2 & 27 & -17 & -17 & 20 & 2 & -11 & 2 & 2 & -1 \\
0 & 0 & 1 & -3 & 2 & 8 & -17 & 5 & 31 & -41 & -6 & 61 & -31 & -37 & 43 & 7 & -23 & 5 & 6 & -2 & 0 \\
0 & 1 & -3 & 2 & 9 & -19 & 5 & 37 & -46 & -17 & 73 & -28 & -52 & 46 & 14 & -29 & 4 & 9 & -4 & -1 & 1 \\
1 & -3 & 2 & 7 & -17 & 4 & 30 & -38 & -16 & 59 & -21 & -41 & 33 & 8 & -19 & 3 & 4 & -2 & 0 & 0 & 0 \\
-1 & 1 & 2 & -7 & 3 & 13 & -19 & -6 & 29 & -11 & -19 & 17 & 4 & -9 & 2 & 2 & -1 & 0 & 0 & 0 & 0 \\
0 & 1 & -2 & 1 & 4 & -7 & 1 & 9 & -7 & -3 & 6 & -1 & -2 & 1 & 0 & 0 & 0 & 0 & 0 & 0 & 0
\end{array}\right)
$$


$H_{[2]}^{10_{108}}=\frac{1}{q^{20} A^{4}}\left(\begin{array}{ccccccccccccccccccccc}0 & 0 & 0 & 0 & 0 & 0 & 0 & 1 & -2 & -1 & 5 & -2 & -5 & 5 & 1 & -4 & 3 & 0 & -2 & 1 & 0 \\ 0 & 0 & 0 & 0 & -1 & 1 & 4 & -6 & -6 & 14 & 3 & -19 & 5 & 14 & -11 & -1 & 6 & -5 & 1 & 2 & -1 \\ 0 & 0 & 1 & -3 & 0 & 11 & -9 & -17 & 25 & 12 & -35 & 5 & 27 & -17 & -5 & 11 & -7 & 0 & 3 & -2 & 0 \\ 0 & 1 & -3 & -1 & 12 & -7 & -21 & 23 & 17 & -36 & 1 & 29 & -17 & -7 & 15 & -8 & -3 & 7 & -2 & -1 & 1 \\ 1 & -3 & -1 & 11 & -7 & -17 & 21 & 10 & -28 & 6 & 19 & -15 & 0 & 10 & -8 & 0 & 4 & -2 & 0 & 0 & 0 \\ -1 & 1 & 5 & -5 & -7 & 13 & 3 & -16 & 6 & 10 & -11 & 1 & 6 & -6 & 0 & 2 & -1 & 0 & 0 & 0 & 0 \\ 0 & 1 & -2 & -2 & 5 & -1 & -6 & 5 & 2 & -5 & 3 & 1 & -2 & 1 & 0 & 0 & 0 & 0 & 0 & 0 & 0\end{array}\right)$

$H_{[2]}^{10_{111}}=\frac{A^{4}}{q^{14}}\left(\begin{array}{ccccccccccccccccccccc}0 & 0 & 0 & 0 & 0 & 0 & 0 & 1 & -2 & -1 & 6 & -1 & -7 & 5 & 3 & -5 & 2 & 1 & -2 & 1 & 0 \\ 0 & 0 & 0 & 0 & -1 & 2 & 3 & -11 & -3 & 22 & -8 & -26 & 20 & 10 & -21 & 6 & 6 & -7 & 2 & 1 & -1 \\ 0 & 0 & 0 & -3 & 5 & 10 & -22 & -9 & 49 & -12 & -51 & 45 & 22 & -43 & 16 & 16 & -16 & 4 & 4 & -3 & 1 \\ 1 & 0 & -6 & 7 & 17 & -29 & -17 & 59 & -11 & -65 & 47 & 25 & -55 & 15 & 20 & -24 & 4 & 6 & -5 & 1 & 0 \\ 0 & -5 & 3 & 15 & -22 & -18 & 46 & -5 & -52 & 36 & 22 & -42 & 14 & 18 & -19 & 5 & 5 & -4 & 1 & 0 & 0 \\ -1 & 2 & 5 & -9 & -5 & 21 & -4 & -22 & 19 & 9 & -20 & 7 & 8 & -8 & 1 & 2 & -1 & 0 & 0 & 0 & 0 \\ 0 & 1 & -2 & -1 & 6 & -3 & -6 & 8 & 0 & -6 & 4 & 1 & -2 & 1 & 0 & 0 & 0 & 0 & 0 & 0 & 0\end{array}\right)$

$H_{[2]}^{10_{113}}=\frac{1}{q^{16}}\left(\begin{array}{ccccccccccccccccccccc}0 & 0 & 0 & 0 & 0 & 0 & 0 & 1 & -4 & 1 & 13 & -15 & -10 & 27 & -6 & -17 & 12 & 2 & -4 & 1 & 0 \\ 0 & 0 & 0 & 0 & -1 & 4 & 2 & -21 & 15 & 38 & -61 & -19 & 83 & -29 & -51 & 40 & 8 & -19 & 3 & 3 & -1 \\ 0 & 0 & 0 & -4 & 9 & 7 & -44 & 30 & 77 & -108 & -32 & 152 & -52 & -89 & 82 & 11 & -41 & 15 & 6 & -5 & 1 \\ 1 & -1 & -7 & 16 & 7 & -62 & 36 & 94 & -132 & -48 & 172 & -59 & -107 & 92 & 10 & -48 & 17 & 6 & -6 & 1 & 0 \\ 0 & -3 & 10 & 6 & -44 & 23 & 81 & -94 & -49 & 139 & -33 & -88 & 71 & 12 & -36 & 12 & 5 & -4 & 1 & 0 & 0 \\ -1 & 3 & 2 & -18 & 9 & 36 & -41 & -25 & 62 & -12 & -40 & 28 & 7 & -14 & 3 & 2 & -1 & 0 & 0 & 0 & 0 \\ 0 & 1 & -3 & 0 & 9 & -8 & -8 & 15 & -2 & -10 & 7 & 1 & -3 & 1 & 0 & 0 & 0 & 0 & 0 & 0 & 0\end{array}\right)$ 


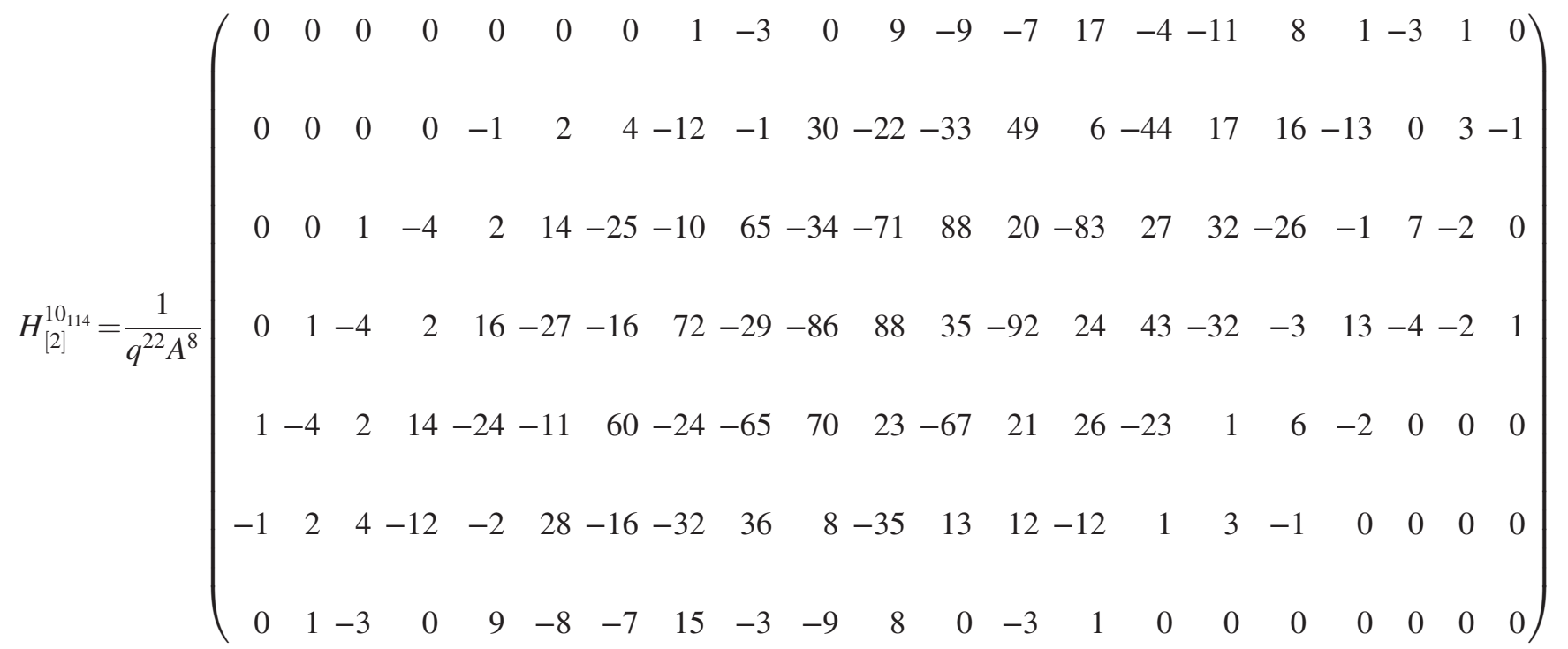

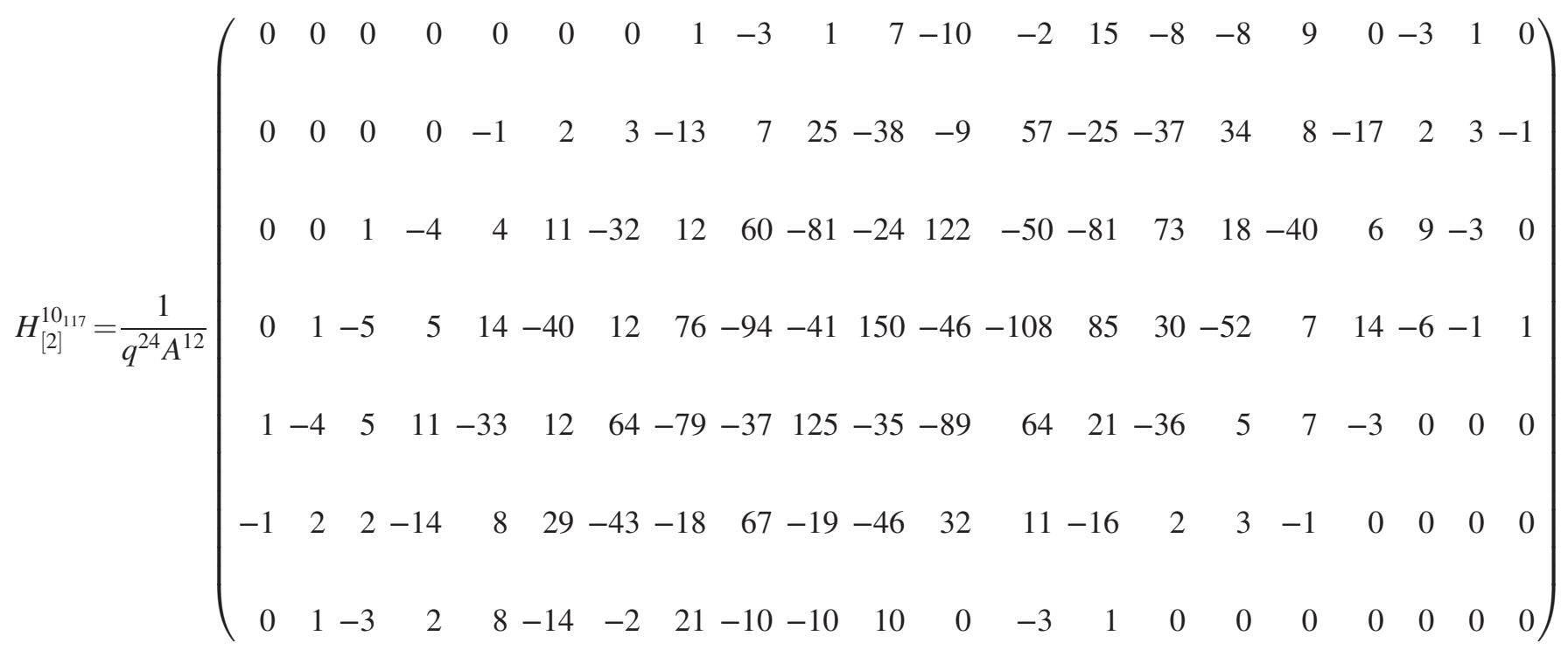

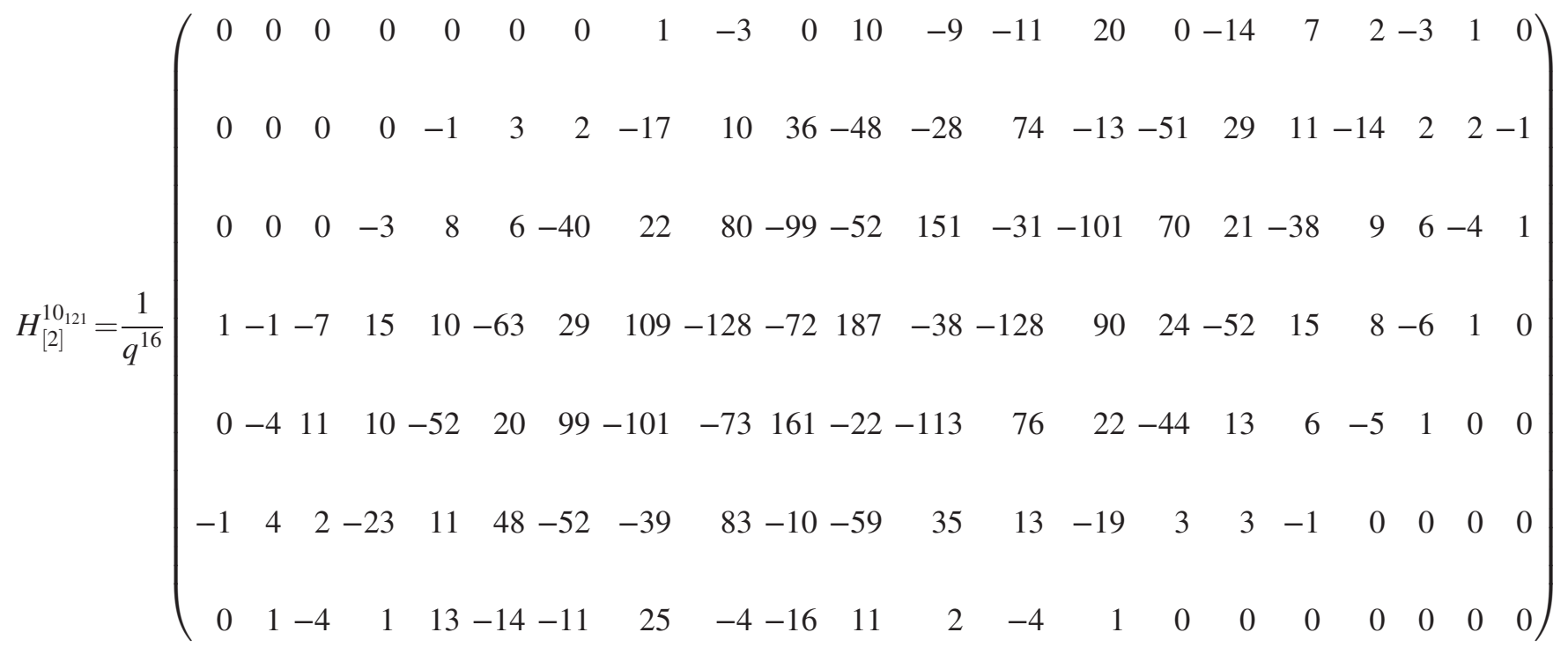




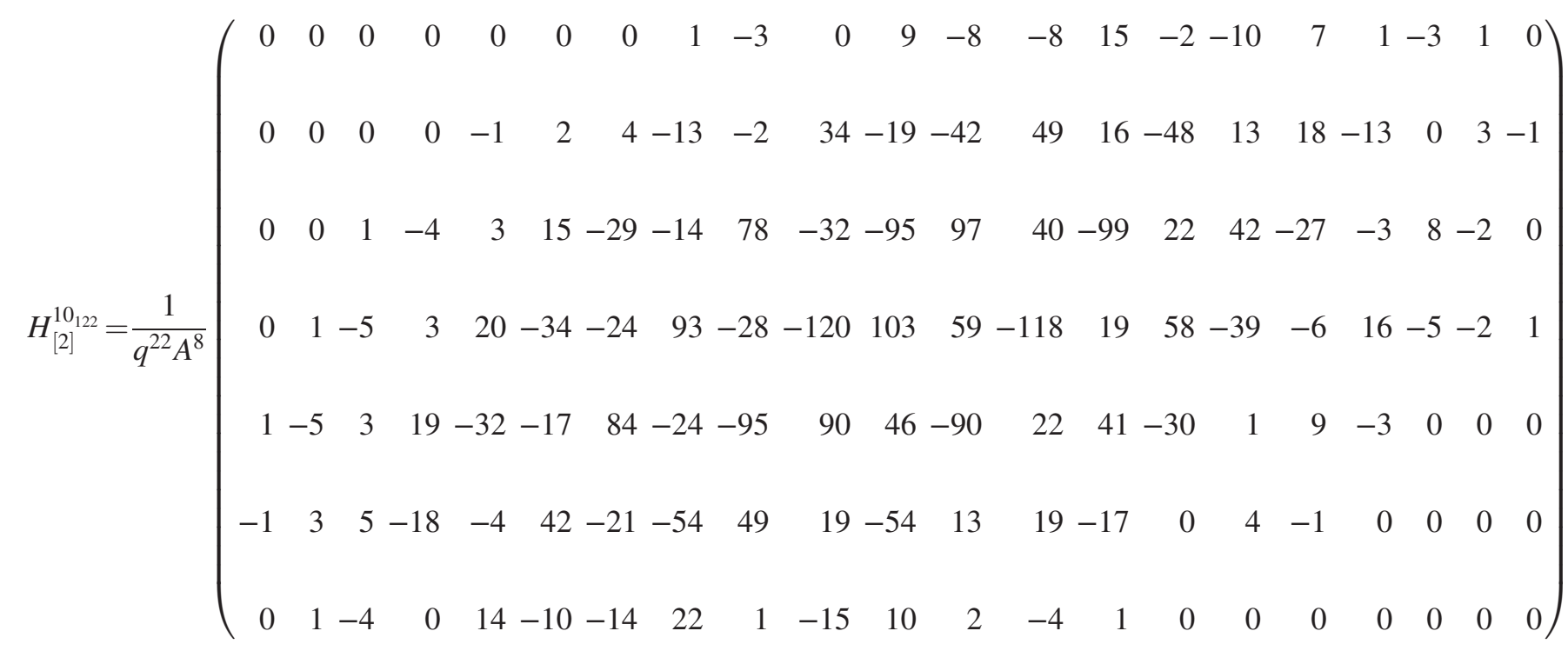

$$
H_{[2]}^{10_{156}}=\frac{1}{q^{20} A^{10}}\left(\begin{array}{cccccccccccccccccccc}
0 & 0 & 0 & 0 & 0 & 1 & -2 & 0 & 3 & -4 & 1 & 5 & -5 & -2 & 5 & -1 & -2 & 1 & 0 \\
0 & 0 & -1 & 1 & 2 & -5 & 3 & 4 & -10 & 6 & 9 & -13 & -2 & 12 & -3 & -6 & 3 & 1 & -1 \\
1 & -2 & 1 & 4 & -7 & 4 & 7 & -16 & 8 & 16 & -20 & -4 & 19 & -5 & -8 & 6 & 1 & -2 & 1 \\
-1 & 1 & 1 & -5 & 4 & 4 & -14 & 6 & 14 & -16 & -5 & 13 & -2 & -6 & 2 & 1 & -1 & 0 & 0 \\
0 & 1 & -1 & 1 & 2 & -6 & 3 & 7 & -8 & -2 & 6 & -1 & -2 & 1 & 0 & 0 & 0 & 0 & 0 \\
0 & 0 & 0 & -1 & 0 & 2 & -1 & -1 & 1 & 0 & 0 & 0 & 0 & 0 & 0 & 0 & 0 & 0 & 0
\end{array}\right)
$$$$
H_{[2]}^{10_{158}}=\frac{1}{q^{18} A^{4}}\left(\begin{array}{ccccccccccccccccccc}
0 & 0 & 0 & 0 & 0 & 0 & 0 & 0 & 0 & 0 & 0 & 0 & 0 & 1 & 0 & 0 & 0 & 0 & 0 \\
0 & 0 & 0 & 0 & 0 & 0 & 0 & 0 & 0 & 1 & -2 & -1 & 4 & -1 & -3 & 1 & 1 & 0 & 0 \\
0 & 0 & 0 & 0 & 0 & 1 & -3 & 1 & 7 & -12 & -2 & 18 & -9 & -11 & 9 & 1 & -4 & 0 & 0 \\
0 & 0 & -1 & 1 & 2 & -8 & 5 & 14 & -24 & -2 & 34 & -16 & -19 & 20 & 3 & -9 & 3 & 2 & -1 \\
1 & -2 & 2 & 5 & -11 & 5 & 21 & -29 & -8 & 43 & -17 & -25 & 24 & 1 & -11 & 5 & 1 & -2 & 1 \\
-1 & 1 & 1 & -8 & 3 & 13 & -20 & -9 & 29 & -9 & -19 & 14 & 2 & -7 & 2 & 1 & -1 & 0 & 0 \\
0 & 1 & -2 & 1 & 6 & -7 & -3 & 13 & -4 & -6 & 6 & 0 & -2 & 1 & 0 & 0 & 0 & 0 & 0
\end{array}\right)
$$ 


\begin{tabular}{|c|c|c|c|c|c|c|c|c|c|c|c|c|c|c|c|c|c|c|c|}
\hline & 0 & 0 & 0 & 0 & 0 & 0 & 0 & 0 & 0 & 0 & 0 & 0 & 0 & 0 & 1 & 0 & 0 & 0 & $0)$ \\
\hline & 0 & 0 & 0 & 0 & 0 & 0 & 0 & 0 & 0 & -1 & 1 & 1 & -3 & 1 & 2 & -2 & -1 & 0 & 0 \\
\hline & 0 & 0 & 0 & 0 & 0 & 1 & -1 & -2 & 1 & 2 & -2 & -3 & 3 & 2 & -1 & -1 & 0 & 2 & 0 \\
\hline$H_{[2]}^{10_{160}}=\frac{A^{4}}{q^{14}}$ & 0 & 0 & -1 & 1 & 3 & -2 & -3 & 3 & 4 & -4 & -4 & 5 & 1 & -3 & -1 & -1 & 1 & 0 & -1 \\
\hline & 1 & -2 & -1 & 5 & -2 & -6 & 4 & 4 & -5 & -2 & 4 & 1 & 0 & 0 & -2 & 3 & 1 & -2 & 1 \\
\hline & -1 & 0 & 3 & -1 & -3 & 3 & 2 & -3 & -1 & 2 & 0 & 0 & 0 & -2 & 1 & 1 & -1 & 0 & 0 \\
\hline & 0 & 1 & -1 & -1 & 2 & 0 & -2 & 1 & 1 & -1 & 1 & 0 & -1 & 1 & 0 & 0 & 0 & 0 & 0 \\
\hline
\end{tabular}

$$
\begin{aligned}
& \left(\begin{array}{rrrrrrrrrrrrrrrrrrrrrrrrrrrr}
0 & 0 & 0 & 0 & 0 & 0 & 0 & 3 & -1 & 0 & 5 & 0 & -2 & 3 & 1 & 0 \\
0 & 0 & 0 & 0 & -3 & 1 & 3 & -9 & -1 & 8 & -9 & -8 & 5 & 0 & -4 & -1
\end{array}\right) \\
& \begin{array}{llllllllllllllll}
0 & 0 & 2 & -5 & 3 & 9 & -15 & 1 & 22 & -13 & -11 & 16 & 3 & -6 & 2 & 1
\end{array} \\
& H_{[2]}^{10_{162}}=\frac{1}{q^{18} A^{12}} \quad \begin{array}{lllllllllllllllllll}
1 & 1 & -5 & 4 & 10 & -16 & -1 & 25 & -11 & -15 & 14 & 4 & -6 & 0 & 1 & 0
\end{array} \\
& \begin{array}{llllllllllllllll}
0 & -3 & 2 & 6 & -12 & -3 & 17 & -8 & -12 & 8 & 2 & -3 & 0 & 0 & 0 & 0
\end{array} \\
& \begin{array}{llllllllllllllll}
-1 & 1 & 2 & -5 & 0 & 7 & -3 & -4 & 3 & 1 & -1 & 0 & 0 & 0 & 0 & 0
\end{array} \\
& \begin{array}{llllllllllllllll}
0 & 1 & -1 & 0 & 2 & -1 & -1 & 1 & 0 & 0 & 0 & 0 & 0 & 0 & 0 & 0
\end{array}
\end{aligned}
$$

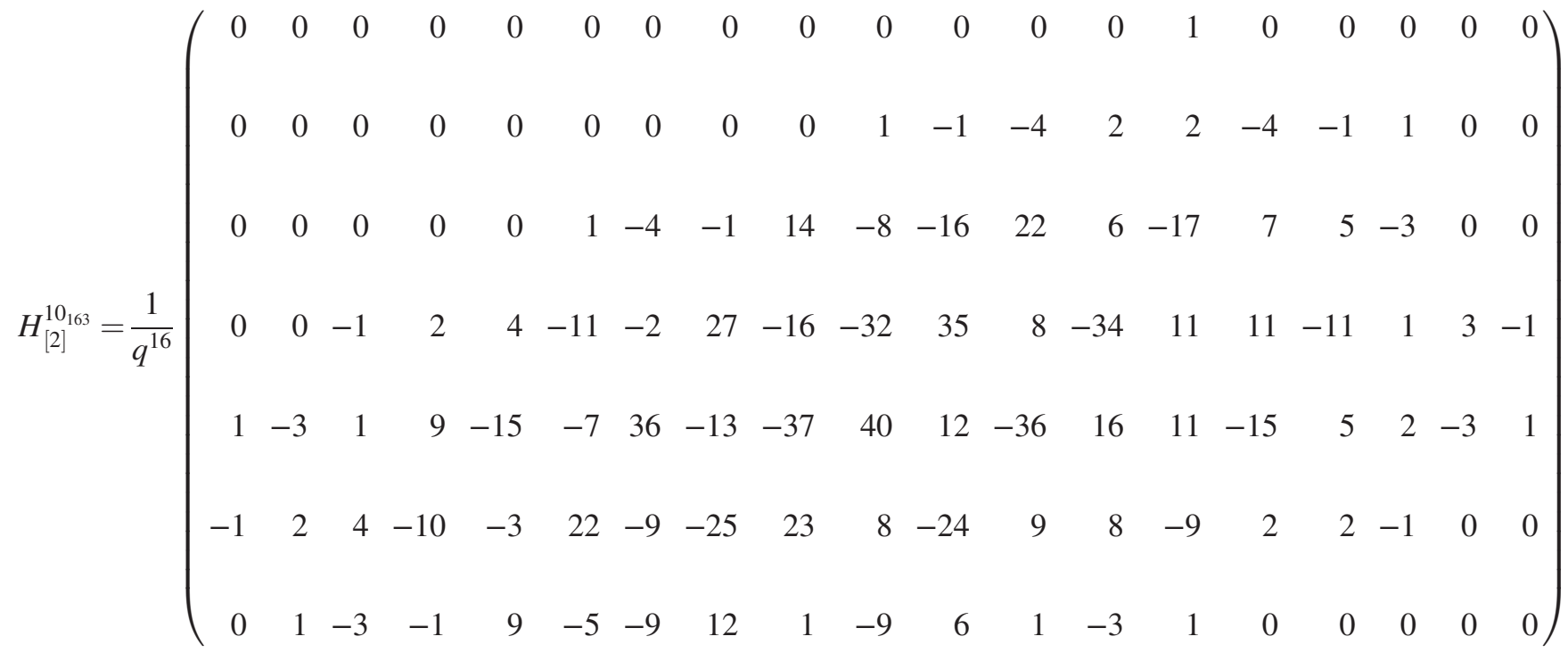




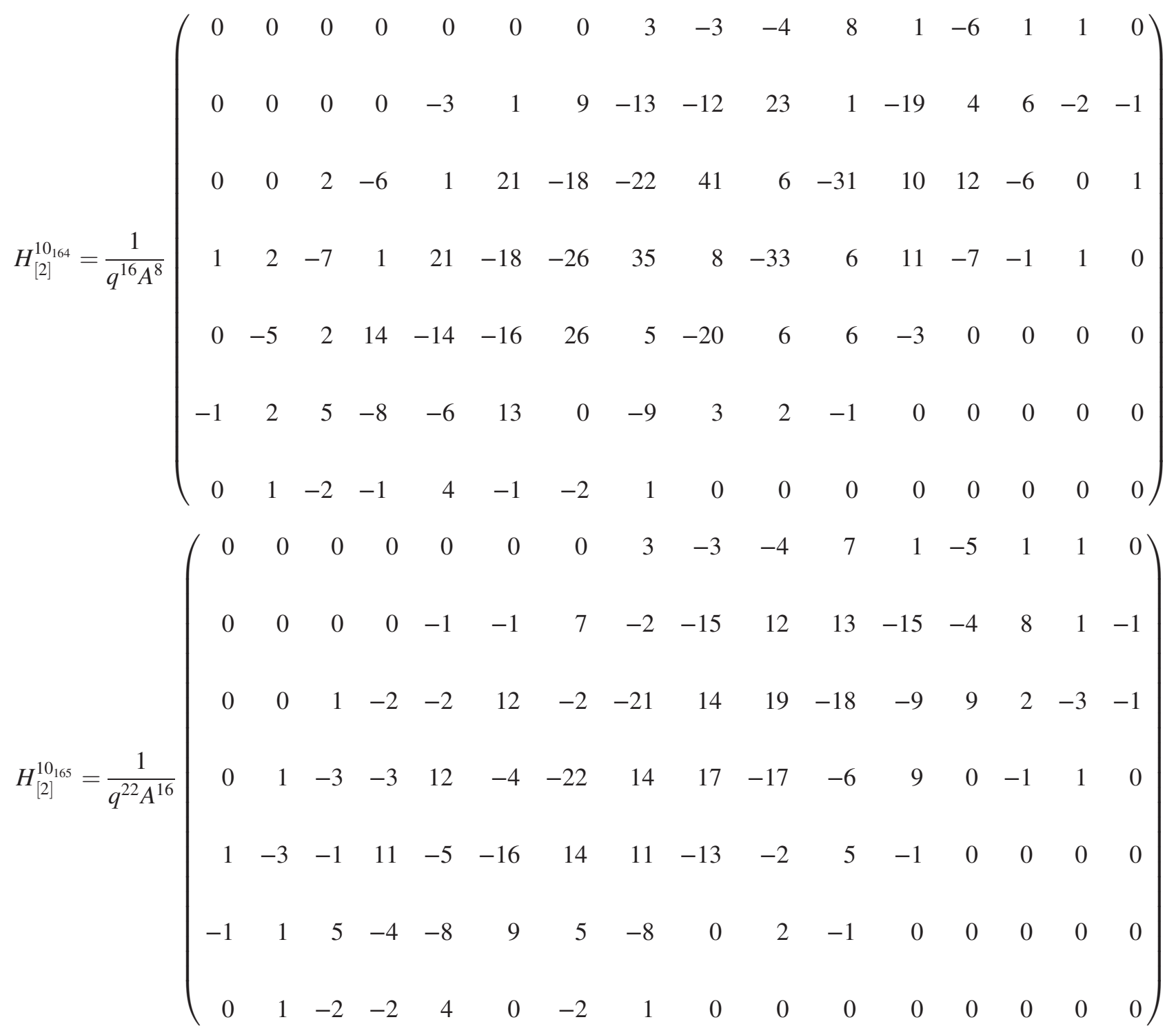

[1] J. W. Alexander, Trans. Am. Math. Soc. 30, 275 (1928); V. F. R. Jones, Inventiones Mathematicae 72, 1 (1983); Bull. Am. Math. Soc. 12, 103 (1985); Ann. Math. (N. Y.) 126, 335 (1987); L. Kauffman, Topology 26, 395 (1987); P. Freyd, D. Yetter, J. Hoste, W. B. R. Lickorish, K. Millet, and A. Ocneanu, Bull. Am. Math. Soc. 12, 239 (1985); J. H. Przytycki and K. P. Traczyk, J. Math. 4, 115 (1987).

[2] J.H. Conway, in Computational Problems in Abstract Algebra, Proc. Conf. Oxford, 1967, edited by J. Leech (Pergamon Press, New York, 1970), p. 329.

[3] E. Witten, Commun. Math. Phys. 121, 351 (1989).

[4] S.-S. Chern and J. Simons, Ann. Math. (N. Y.) 99, 48 (1974).

[5] L. D. Landau and E. M. Lifshitz, Quantum Mechanics: NonRelativistic Theory, 3rd ed. (Pergamon, New York, 1977); A. N. Kirillov and N. Yu. Reshetikhin, in Representations of the Algebra Uq(2), q-Orthogonal Polynomials and Invariants of Links, edited by T. Kohno, New developments in the theory of knots, pp. 202-256; L. Alvarez-Gaune, C. Gomez, and S. Sierra, Phys. Lett. B 220, 142 (1989); J. S. Carter, D. E. Flath, and M. Saito, The Classical and Quantum 6j-symbols (Princeton University Press, Princeton, NJ, 1995).

[6] S. Nawata, P. Ramadevi, and Zodinmawia, J. Knot Theory Ramif. 22, 1350078 (2013).

[7] A. Mironov, A. Morozov, and A. Sleptsov, J. High Energy Phys. 07 (2015) 069.

[8] P. Dunin-Barkowski, A. Mironov, A. Morozov, A. Sleptsov, and A. Smirnov, J. High Energy Phys. 03 (2013) 021.

[9] A. Mironov, A. Morozov, and An. Morozov, AIP Conf. Proc. 1562, 123 (2013). 
[10] S. Arthamonov, A. Mironov, A. Morozov, and An. Morozov, J. High Energy Phys., 04 (2014) 156.

[11] S. Nawata, P. Ramadevi, and Zodinmawia, Lett. Math. Phys. 103, 1389 (2013).

[12] A. Mironov, A. Morozov, and A. Sleptsov, Pis'ma Zh. Eksp. Teor. Fiz. 106, 607 (2017) [JETP Lett. 106, 630 (2017)].

[13] A. Caudron, Classification des noeuds et des enlacements, Publ. Math. Orsay 82-4, University of Paris XI, Orsay, 1982; F. Bonahon and L. C. Siebenmann, New geometric splittings of classical knots and the classification and symmetries of arborescent knots, http://www-bcf.usc.edu/ $\sim$ fbonahon/Research/Preprints/BonSieb.pdf, 2010.

[14] H. Itoyama, A. Mironov, A. Morozov, and An. Morozov, J. High Energy Phys. 07 (2012) 131.

[15] P. Ramadevi, T. R. Govindarajan, and R. K. Kaul, Mod. Phys. Lett. A 09, 3205 (1994); D. Galakhov, D. Melnikov, A. Mironov, A. Morozov, and A. Sleptsov, Phys. Lett. B 743, 71 (2015); Zodinmawia's Ph.D. thesis, 2014; S. Nawata, P. Ramadevi, and V. K. Singh, J. Knot Theor. Ramifications 26, 1750096 (2017).

[16] A. Mironov, A. Morozov, An. Morozov, and A. Sleptsov, JETP Lett. 104, 56 (2016); A. Morozov, Nucl. Phys. B911, 582 (2016); J. High Energy Phys. 09 (2016) 135; Mod. Phys. Lett. A 33, 1850062 (2018); Phys. Lett. B 766, 291 (2017).

[17] J. Gu and H. Jockers, Commun. Math. Phys. 338, 393 (2015); A. Anokhina, A. Mironov, A. Morozov, and An. Morozov, Nucl. Phys. B882, 171 (2014); A. Mironov, A. Morozov, and An. Morozov, Mod. Phys. Lett. A29, 1450183 (2014); A. Morozov, Phys. Lett. B 766, 291 (2017).

[18] A. Mironov, A. Morozov, An. Morozov, P. Ramadevi, and V. K. Singh, J. High Energy Phys. 07 (2015) 109.

[19] E. Guadagnini, M. Martellini, and M. Mintchev, ChernSimons field theory and quantum groups, in Clausthal 1989, Proceedings of quantum groups, pp. 307-317; Phys. Lett. B 235, 275 (1990); N. Yu. Reshetikhin and V. G. Turaev, Commun. Math. Phys. 127, 1 (1990).

[20] A. Anokhina, A. Mironov, A. Morozov, and An. Morozov, Adv. High Energy Phys. 2013 (2013) 931830.

[21] A. Mironov, A. Morozov, and An. Morozov, J. High Energy Phys. 03 (2012) 034.

[22] H. Itoyama, A. Mironov, A. Morozov, and An. Morozov, Int. J. Mod. Phys. A 27, 1250099 (2012).

[23] A. Mironov, A. Morozov, An. Morozov, and A. Sleptsov, Int. J. Mod. Phys. A 30, 1550169 (2015).
[24] A. Mironov, A. Morozov, An. Morozov, and A. Sleptsov, J. High Energy Phys. 09 (2016) 134.

[25] Sh. Shakirov and A. Sleptsov, arXiv:1611.03797.

[26] A. Mironov and A. Morozov, Nucl. Phys. B899, 395 (2015).

[27] A. Mironov, A. Morozov, An. Morozov, P. Ramadevi, V. K. Singh, and A. Sleptsov, J. Phys. A 50, 085201 (2017).

[28] H. Itoyama, A. Mironov, A. Morozov, and An. Morozov, Int. J. Mod. Phys. A 28, 1340009 (2013).

[29] A. Mironov and A. Morozov, Phys. Lett. B 755, 47 (2016).

[30] P. Vogel, The Universal Lie Algebra, http://webusers .imj-prg.fr/ pierre.vogel/.

[31] A. Mironov, R. Mkrtchyan, and A. Morozov, J. High Energy Phys. 02 (2016) 78.

[32] A. Mironov and A. Morozov, Eur. Phys. J. C 78, 284 (2018).

[33] I. Tuba and H. Wenzl, arXiv:math/9912013

[34] Database of colored knot polynomials and supplementary materials (Racah matrices etc.), http://www.knotebook.org.

[35] R. K. Kaul and T. R. Govindarajan, Nucl. Phys. B380, 293 (1992); P. Ramadevi, T. R. Govindarajan, and R. K. Kaul, Nucl. Phys. B402, 548 (1993); B422, 291 (1994); P. Ramadevi and T. Sarkar, Nucl. Phys. B600, 487 (2001).

[36] A. Mironov, A. Morozov, and An. Morozov, in Strings, Gauge Fields, and the Geometry Behind: The Legacy of Maximilian Kreuzer, edited by A. Rebhan, L. Katzarkov, J. Knapp, R. Rashkov, and E. Scheidegger, (World Scientific, Singapore, 2013), p. 101; A. Anokhina, A. Mironov, A. Morozov, and An. Morozov, Nucl. Phys. B868, 271 (2013); A. Anokhina, arXiv:1412.8444; A. Mironov, A. Morozov, An. Morozov, and A. Sleptsov, Phys. Lett. B 760, 45 (2016).

[37] N. Yu. Reshetikhin, L. A. Takhtadjan, and L. D. Faddeev, Algebra and Analysis 1, 178 (1989).

[38] M. Rosso and V. F. R. Jones, Knot Theory Ramifications 02 , 97 (1993); M. Tierz, Mod. Phys. Lett. A 19, 1365 (2004); X.-S. Lin and H. Zheng, Trans. Am. Math. Soc. 362, 1 (2010); A. Brini, B. Eynard, and M. Mariño, Ann. Henri Poincaré 13, 1873 (2012).

[39] S. Nawata, P. Ramadevi, Zodinmawia, and X. Sun, J. High Energy Phys. 11 (2012) 157.

[40] D. Bar-Natan and S. Morrison, Database of various knot invariants mostly uncolored, http://katlas.org.

[41] A. Anokhina and An. Morozov, Theor. Math. Phys. 178, 1 (2014). 